Article

\title{
Evaluating Change of Marginal Bone Height with Cone-Beam Computed Tomography Following Surgical Treatment with Guided Tissue Regeneration (Bone Grafting) or Access Flap Alone: A Retrospective Study
}

\author{
In-Kyung Lee ${ }^{1,+}$, Hyun-Seok Choi ${ }^{1}$, Sang-Heon Jeong ${ }^{2}$ and Jung-Tae Lee ${ }^{3, *,+} \mathbb{D}$ \\ 1 Department of Periodontics, Jukjeon Dental Hospital, College of Dentistry, Dankook University, \\ Yongin 16890, Korea; perio8296@dankook.ac.kr (I.-K.L.); futurechoi0621@gmail.com (H.-S.C.) \\ 2 Department of Radiology, Jukjeon Dental Hospital, College of Dentistry, Dankook University, \\ Yongin 16890, Korea; serch34@hanmail.net \\ 3 Department of Periodontics, One-Stop Specialty Center, Seoul National University, Dental Hospital, \\ Seoul 05698, Korea \\ * Correspondence: jungtae1308@hanmail.net; Tel.: +82-2-2072-0054; Fax: +82-2-2072-3018 \\ + These authors contributed equally to the study.
}

check for

updates

Citation: Lee, I.-K.; Choi, H.-S.; Jeong, S.-H.; Lee, J.-T. Evaluating Change of Marginal Bone Height with Cone-Beam Computed Tomography Following Surgical Treatment with Guided Tissue Regeneration (Bone Grafting) or Access Flap Alone: A Retrospective Study. Medicina 2021, 57, 869. https://doi.org/10.3390/ medicina57090869

Academic Editor: Gaetano Isola

Received: 26 July 2021

Accepted: 21 August 2021

Published: 25 August 2021

Publisher's Note: MDPI stays neutral with regard to jurisdictional claims in published maps and institutional affiliations.

Copyright: (c) 2021 by the authors. Licensee MDPI, Basel, Switzerland. This article is an open access article distributed under the terms and conditions of the Creative Commons Attribution (CC BY) license (https:/ / creativecommons.org/licenses/by/ $4.0 /)$.

\begin{abstract}
Background and Objectives: This study aimed to evaluate the change of bone height following treatment of human intrabony defects with guided tissue regeneration (GTR) with bone grafting or access flap alone by cone-beam computed tomography (CBCT) scan. Materials and methods: This study was conducted as a retrospective longitudinal study. In this study, a total of 2281 teeth sites were included: the GTR group had 1210 sites, and the Flap group had 1071 sites. In the GTR group, demineralized freeze-dried bone (DFDBA) particles in combination with resorbable collagen membrane were used. No regenerative material was applied to the Flap group. CBCT images were taken twice at baseline and at least 2.5 months postoperatively. Bone heights were measured using software on CBCT images. Results: The bony change between the GTR and Flap groups was significantly different $(p=0.00001)$. Both males and females in the GTR group had smaller bone loss than in the Flap group. In age groups, significant differences of bony height between the GTR and Flap groups were observed in the subgroups consisting of those $29-45$ and $46-53$ years old. The non-smoking subjects in the GTR group had higher bone heights than those in the Flap group. In the absence of systemic disease and medicine, bone formation was higher in the GTR group than in the Flap group. In terms of oral position, the \#14-17, \#34-37, and \#44-47 subgroups of the GTR group showed higher levels of bone heights than those of the Flap group. Conclusions. The results of this study indicated that the GTR procedure offers the additional benefit of higher bone heights than the Flap procedure does.
\end{abstract}

Keywords: periodontitis; dental materials; guided tissue regeneration; osseous defects; periodontal regeneration; periodontal surgery

\section{Introduction}

The main objective of periodontal surgery is to contribute to the long-term preservation of the periodontium by facilitating plaque removal and infection control. Periodontal surgery is divided into open flap surgery and guided tissue regeneration (GTR). Open flap surgery aims to reconstruct the periodontal pocket by removing inflamed tissue and subgingival calculus. Open flap surgery provides the following: (1) accessibility for proper professional scaling and root planing; (2) establishing a gingival morphology that facilitates self-performed infection control; and (3) creating new attachment in the destructive site of the gingiva [1]. GTR is the regenerative procedure using bone graft material and barrier membranes [2,3]. GTR has the advantage of mechanical reinforcement of bony 
defects with bone graft material and membranes, thus providing space maintenance during healing periods. In the previous study, GTR has been shown to result in significantly more periodontal regeneration [4]. Sculean et al. and Stavropoulos et al. investigated that GTR had more increased clinical attachment level (CAL) gains compared with flap alone in clinical studies $[5,6]$. These approaches (GTR or open flap surgery), while offering tangible benefits, remain inconclusive. Few cases did enough to provide evidence of which procedure is better.

Demineralized freeze-dried bone (DFDBA) particles have been used in combination with barrier membrane in GTR, resulting in enhanced periodontal regeneration due to the osteoinductive ability of DFDBA to stimulate bone formation. DFDBA is associated with the amount of bone morphogenic proteins that remain after the demineralization process is completed $[7,8]$. A previous study demonstrated that probing pocket depth (PPD) and CAL gain were observed in sites treated with a combination of DFDBA and barrier membrane [9]. Similarly, Kiany and Moloudi reported favorable results after using DFDBA and barrier membrane [10].

A barrier membrane should have five characteristics to support ideal function: biocompatibility, space-maintenance ability, tissue integration, cell occlusiveness, and ease of manipulation [11]. Resorbable membrane has been used in GTR due to its biocompatibility [12]. However, resorbable membrane has weak physical properties. To maintain infrabony defect with bone graft material, resorbable membrane needs to be strengthened. A cross-linking procedure has been introduced that increases mechanical property and slows the degradation of resorbable membranes [13]. A cross-linking technique of resorbable membranes has two classified methods: physical and chemical methods. Physically cross-linked membranes were manufactured using ultraviolet (UV) irradiation or a dehydrothermal (DHT) technique. Chemically cross-linked membranes were made by glutaraldehyde (GTA) and hexamethylene diisocyanate (HMDI) [14]. A previous study demonstrated that cross-linked membranes overcome a shortage of non-cross-linked membranes [15].

To gain accurate results of periodontal treatment, various measurement methods have been developed. Clinical methods such as bone sounding are not always able to provide accurate tissue measurement. Surgical reentry and histologic evaluation are prohibited due to ethical issues. Therefore, radiographic methods (intraoral periapical and panoramic radiographs) were used to evaluate differences in marginal bone loss before and after surgery. The limitation of conventional radiographic measurement is that it cannot provide multi-dimensional assessment. Cone-beam computed tomography (CBCT), a reliable and widely used tool, provides three-dimensional exploration of periodontal osseous defects and assesses the furcation area of molars [16-18].

The aim of this study was to evaluate the change of bone height following treatment of human intrabony defects with GTR (bone grafting) or access flap alone.

\section{Materials and Methods}

\subsection{Study Population}

The study was conducted as a retrospective longitudinal study. The study was conducted according to the guidelines of the Declaration of Helsinki and approved by the Institutional Review Board for Clinical Research at Dankook University College of Dentistry Jukjeon Dental Hospital (approval no. 201910-001-002). From May 2015 to May 2018, 178 patients with 2520 sites (GTR: 97 patients (1296 sites); Flap: 81 patients (1224 sites)) who visited the department of periodontics at Dankook University College of dentistry Jukjeon Dental Hospital for periodontal treatment were selected. All patients met the inclusion criteria as follows by referring to previous studies [19-21].

- Clinical and radiographical diagnosis of severe chronic periodontitis;

- Presence of at least one intrabony defect of $\geq 3 \mathrm{~mm}$ on the radiographs;

- $\quad P P D \geq 5 \mathrm{~mm}$ in the intrabony defects. 
Written informed consent was obtained from all the patients. Each site designated mesial and distal aspects on a panoramic view of CBCT images. After sorting due to scattering of CBCT image and extraction (28 teeth (56 sites) in Flap group) during the follow-up period, we divided the 2 groups by site (GTR: 1210 sites and Flap: 1071 sites). Premolars and molars of maxilla and mandible were included. Study participants were excluded if they did not complete periodontal treatment, had teeth extracted during the follow-up period, had no CBCT images both before and after treatment, and had $<5 \mathrm{~mm}$ PPD, which is not suitable for periodontal surgery (GTR or Flap) (Figure 1). Sample size calculation in this study referenced previous similar research by Tonetti et al. [20]. The power of 0.8 and an alpha level of 0.05 were used to detect the difference between groups.

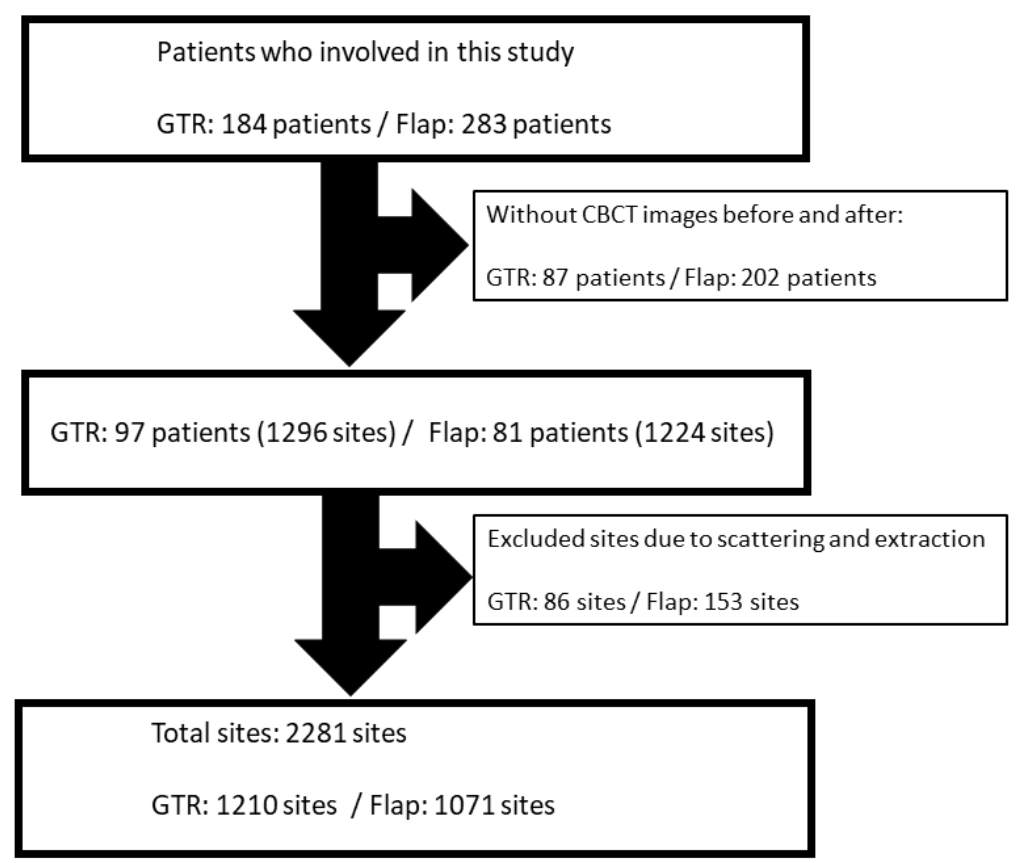

Figure 1. A flow diagram of study participants' enrollment and follow-up. GTR: guided tissue regeneration; CBCT: cone-beam computed tomography.

\subsection{Pre-Treatment}

Scaling or root planing was performed on all participants prior to the experimental phase.

\subsection{Surgical Procedures}

One periodontist (JTL) performed all surgical procedures. The patients received oral hygiene instructions before periodontal surgery. Under local anesthesia using $2 \%$ lidocaine (1:100,000 epinephrine, Huons Co., Ltd., Sungnam, Korea), the surgical sites were treated. In the GTR group, a full thickness flap was performed. The exposed defects were carefully scaled and root planed using a combination of mechanical and hand instruments. DFDBA (Human Cortical Powder, Demineralised, DIZG, Berlin, Germany) was applied to the defect area. A cross-linked-collagen membrane (OssGuide, Hyundai bioland Co., Ltd., Cheongju, Korea) composed of porcine pericardium-derived type I collagen was used. It was trimmed to the local anatomy and then positioned on the graft material. Then, the flap was replaced and sutured with resorbable sutures (4-0 Vicryl, Ethicon, Somerville, NJ, USA). All patients were prescribed amoxycillin and clavulanic acid $375 \mathrm{mg}$ (Augmentin, Ilsung Pharma Co., Seoul, Korea), naproxen sodium (Anaprox, Jongeun Dang Pharmceutical, Co., Seoul, Korea), and almagate (Almagel, Yuhan Pharma Co., Seoul, Korea) 3 times daily for 1 week unless an allergy to penicillin was present. All patients were instructed to rinse for 30 seconds twice daily with $0.12 \%$ chlorhexidine gluconate (Hexamedin, Bukwang Pharmaceutical, Ansan, Korea) for 1 week. Sutures were removed 2 weeks post-surgery. In the Flap group, full thickness flap, scaling, and root planing were performed. Irregular 
bony protrusion was removed with rotary or hand instruments. After debridement, the flap was repositioned and sutured. The same medicines were prescribed as in the GTR group (Figure 2).
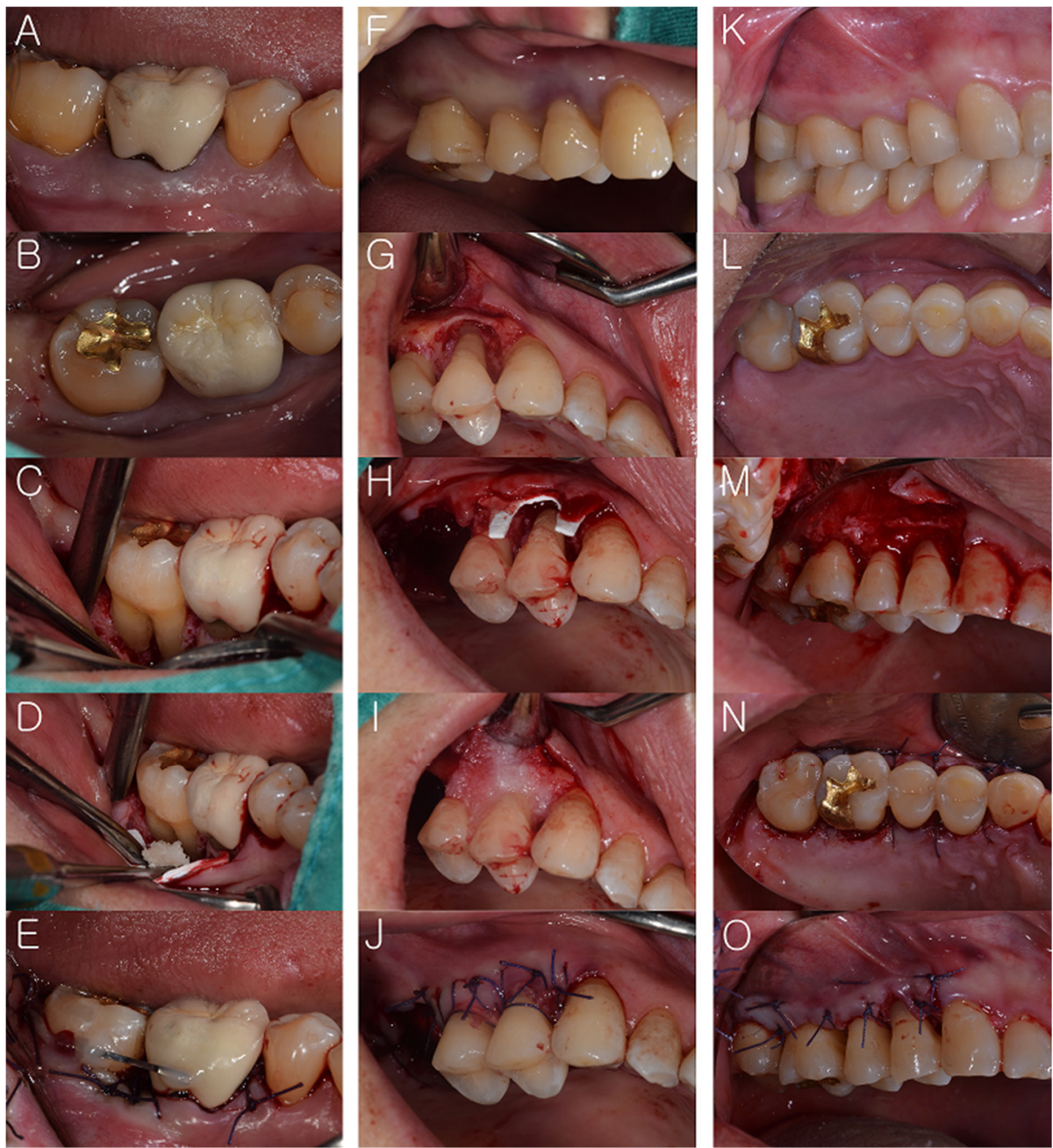

Figure 2. (Case 1: A-E and Case 2: F-J): Guided tissue regeneration (GTR); (A-B,F) before treatment, $(\mathrm{C}, \mathrm{G})$ full thickness flaps were performed; then, the exposed defects were carefully scaled and root planed, (D,H-I) demineralized freeze-dried bone (DFDBA) and collagen membrane were applied to the defect area, $(\mathbf{E}, \mathbf{J})$ suture and resin wire splint were performed. (Case 1: $\mathbf{K}-\mathbf{O}$ ): Open flap surgery (Flap); (K,L) before treatment, (M) full thickness flap and debridement were also performed, (N,O) suture was performed.

\subsection{CBCT Taking and Maintenance Care (3 and 6 Months)}

CBCT images were taken twice at baseline (before periodontal surgery) and at least 2.5 months postoperatively. A supportive care program and professional calculus removal were provided to all patients at 3 and 6 months. After that, regular oral cleaning was performed every 6 months if the patients maintained their oral hygiene. 


\subsection{Radiographic Evaluation and Bone Height Measurement}

A CBCT scanner (Kodak 9500, Carestream Health, Rochester, NY, USA) was used in this study, providing a grayscale image of 14 bits with a voxel size of $0.2 \mathrm{~mm}$ per side. The CBCT images were viewed using 3D imaging software (OnDemand 3D, Cybermed Co., Seoul, Korea). In order to evaluate change of bone height, CBCT images were measured twice before and after treatment on the same site. The cemento-enamel junction (CEJ) was set as an unchanged reference [16]. If an implant was involved, the reference point was the connection between the abutment and the crown. In the preoperative CBCT image, the distance of the CEJ-base of alveolar bone of the mesial and distal sites of the teeth was measured (Figure 3A-D). To overlap the images of the same cross-section as much as possible before and after treatment, imaginary lines were used (Figure 3E; connecting line of CEJ, A, and D: bone height of first CBCT image, Figure 3F: connecting line of basic point between Figure 3A,D). The distance of the same site after treatment was measured on the second CBCT image (Figure $3 \mathrm{~A}^{\prime}-\mathrm{D}^{\prime}$ ). The amount of bony change was confirmed by subtracting the first values from the second values (Figure $3 \mathrm{~A}^{\prime}-\mathrm{D}^{\prime}, \mathrm{A}-\mathrm{D}$ ). To attest the consistency of the measurements, each section of the CBCT image was assigned a serial number. All pre- and postsurgical bone height measurements were carried out by a single examiner (H.S.C.). A second examiner (J.T.L.) also evaluated bony height at the same serial number image.
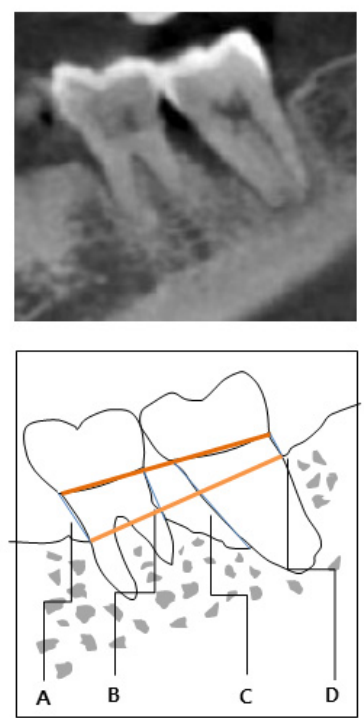

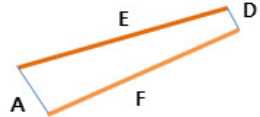

Change of alveolar bone height: Green line - Blue line
$\left(A^{\prime}-A^{\prime} ; B^{\prime}-B, C^{\prime}-C ; D^{\prime}-D\right)$
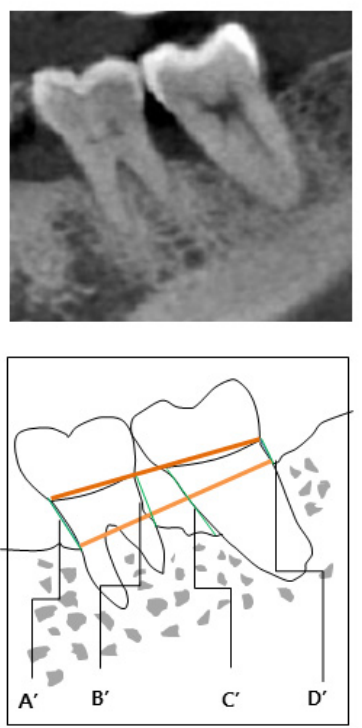

Blue line $-1^{\text {st }}$ measurement of before treatment

A: Bone height of \#36 Mesial (CEJ base of alveolar bone)

B: Bone height of \#36 Distal (CEJ base of alveolar bone)

C: Bone height of \#37 Mesial (CEJ base of alveolar bone)

$\mathrm{D}$ : Bone height of \#37 Distal (CEJ base of alveolar bone)

: Connecting line of CEJ between \#36 mesial and 37 distal point

$\mathrm{F}$ : Connecting line of basic point between $A$ and $D$

Green line $-2^{\text {nd }}$ measurement of after treatment

$A^{\prime}$ : Bone height of \#36 Mesial (CEJ base of alveolar bone) $B^{\prime}$ : Bone height of \#36 Distal (CEJ base of alveolar bone) $C^{\prime}$ : Bone height of \#37 Mesial (CEJ base of alveolar bone) $D^{\prime}$ : Bone height of \#37 Distal (CEJ base of alveolar bone)

Figure 3. (A-D): The distance between the cemento-enamel junction (CEJ) and base of alveolar bone of the mesial and distal sites on the teeth in the preoperative CBCT image, (E): Connecting line of CEJ, (F): Connecting line of basic point between $(\mathbf{A})$ and $(\mathbf{D}),\left(\mathbf{A}^{\prime}-\mathbf{D}^{\prime}\right)$ : The distance of the same site on the second $\mathrm{CBCT}$ image after treatment, $\left(\mathbf{A}^{\prime}-\mathbf{D}^{\prime}\right)$ : The amount of bony change. 


\subsection{Statistical Analysis}

Statistical analysis was performed using SPSS software (SPSS version 23.0, Chicago, IL, USA). To compare variables between GTR and Flap groups, a Mann-Whitney U test was used. Kruskal-Wallis was used to compare variables in 3 or more groups including smoking, systemic disease, medicine, oral regions, and precise site. The threshold for statistical significance was $5 \%$. The post hoc Bonferroni correction was used for multiple comparisons between groups. A logistic regression model was used for multivariable analysis.

\section{Results}

\subsection{Baseline Characteristics}

Baseline demographics are summarized in Table 1. There was a significant different bony change between the GTR and Flap groups $(p=0.00001)$. A total of 1210 subjects of GTR (594 (49.1\%) male and $616(50.9 \%)$ female) and 1071 subjects of Flap (666 (62.2\%) male and $405(37.8 \%)$ female) were included. Both male and female in the GTR group had smaller bone loss than in the Flap group ( $p=0.010$ and $p=0.001$, respectively). The mean age was $49.8 \pm 8.0$ (GTR) and $51.3 \pm 9.4$ (Flap). Significant differences in bony height between GTR and Flap were observed in the 29-45 and 46-53 subgroups ( $p=0.00001$ and $p=0.040$, for each). In GTR group, the 29-45 subgroup had significantly favorable results compared with the 46-53 and 54-76 subgroups ( $p=0.0004$ and $p=0.006$, for each). The non-smoking subjects in the GTR group had higher bone heights than those in the Flap group $(-0.09 \pm 1.13$; GTR and $0.12 \pm 0.93$; Flap, $p=0.009)$. The average intervals between the first and second СBCT was $692.7 \pm 351.3$ days in the GTR group and $688.4 \pm 342.9$ days in the Flap group. All values of CBCT interval were higher in the GTR subgroups than in the subgroups in Flap (87-490 days: $-0.06 \pm 1.23,491-859$ days: $0.02 \pm 1.24: 860-1543$ days: $0.11 \pm 1.40$ vs. $0.17 \pm 0.83,0.20 \pm 1.24,0.20 \pm 0.95$ ). Two subgroups (87-490 and $491-859)$ had significant differences of bone healing in GTR and Flap $(p=0.0001$ and $p=0.018$, respectively). With systemic diseases, there was an association between mean heights of alveolar bone of GTR and Flap in hepatitis. Bone height for the Flap group was higher than in the GTR group with significant difference $(0.32 \pm 0.84$ : GTR vs. $-0.35 \pm 0.74$ : Flap, $p=0.022$ ). No difference was found in other diseases, including hypertension, rhinitis, sinusitis, diabetes mellitus, and hyperlipidemia. In the absence of systemic disease, bone formation was higher in the GTR group than in the Flap group $(0.02 \pm 1.31$ : GTR vs. $0.22 \pm 1.05$ : Flap, $p=0.00001)$. In subjects taking medications for diabetes mellitus and hyperlipidemia, alveolar bone recovery was less in the GTR group than in the Flap group $(0.23 \pm 0.51$ : GTR vs. $-0.21 \pm 0.96$ : Flap, $p=0.018)$. However, there was a significantly higher difference for height of bone in the GTR group without medicine than in the Flap group $(0.03 \pm 1.31$ : GTR vs. $0.21 \pm 1.05$ : Flap, $p=0.00001)$. According to oral position, the \#14-17, \#34-37, and \#44-47 subgroups of the GTR group showed higher levels of bone heights than those of the Flap group $(0.04 \pm 1.21,-0.03 \pm 1.10$, and $-0.03 \pm 1.29$; GTR vs. $0.32 \pm 0.91,0.18 \pm 0.91$, and $0.18 \pm 1.25$; Flap, $p=0.00001,0.024$, and 0.087 , respectively). Only the \#24-27 group had reverse results of other oral sites $(0.10 \pm 1.50$; GTR vs. $0.07 \pm 1.05$; Flap, $p=0.342$ ). In all premolars and molars in the GTR group, bone height was higher than that of the Flap group. In particular, it was significantly higher at \#4, \#5, and \#7 ( $p=0.033,0.002$, and 0.039 , for each) (Figure 4). 
Table 1. Baseline characteristics.

\begin{tabular}{|c|c|c|c|c|c|c|c|}
\hline \multirow{2}{*}{ Variable } & \multicolumn{3}{|c|}{ GTR (mm) } & \multicolumn{3}{|c|}{ Flap (mm) } & \multirow{2}{*}{$p 2$} \\
\hline & $N$ & Mean (SD) & $p 1$ & $N$ & Mean (SD) & $p 1$ & \\
\hline & & 2281 & & & $0.10(1.19)$ & & \\
\hline lotal N/ mean (SD) & 1210 & $0.03(0.13)$ & & 1071 & $0.190(0.104)$ & & $0.00001 *$ \\
\hline Sex & & & 0.939 & & & 0.079 & \\
\hline Male (\%) & $594(49.1)$ & $0.01(1.22)$ & & $666(62.2)$ & $0.18(1.14)$ & & $0.010 *$ \\
\hline Female (\%) & $616(50.9)$ & $0.05(1.37)$ & & $405(37.8)$ & $0.20(0.85)$ & & $0.00002 *$ \\
\hline Age [years, mean (SD)] & & $49.77(8.03)$ & $0.001 *$ & & $51.25(9.40)$ & 0.417 & \\
\hline $29-45(\%)$ & $416(34)$ & $-0.17(1.21)$ & & $376(35)$ & $0.17(0.10)$ & & 0.00001 * \\
\hline $46-53(\%)$ & $388(32)$ & $0.19(1.26)$ & $0.0004 *$ & $312(29)$ & $0.24(1.11)$ & 0.217 & $0.040 *$ \\
\hline $54-76(\%)$ & $406(34)$ & $0.08(1.39)$ & $\begin{array}{c}0.006^{*} \\
0.565\end{array}$ & $383(36)$ & $0.16(1.01)$ & $\begin{array}{l}0.901 \\
0.280\end{array}$ & 0.125 \\
\hline Smoking & & & 0.551 & & & 0.301 & \\
\hline Present $(\%)$ & $280(21.6)$ & $0.10(1.30)$ & & $239(19.5)$ & $0.22(1.23)$ & & 0.120 \\
\hline Past (\%) & $91(7.0)$ & $-0.05(1.05)$ & 0.415 & $82(6.7)$ & $0.09(1.14)$ & 0.218 & 0.693 \\
\hline $\operatorname{Not}(\%)$ & $839(64.7)$ & $0.01(1.32)$ & $\begin{array}{l}0.317 \\
0.930\end{array}$ & $750(61.3)$ & $0.12(0.96)$ & $\begin{array}{l}0.891 \\
0.334\end{array}$ & $0.009 *$ \\
\hline $\begin{array}{c}\text { CBCT interval } \\
\text { [days, mean (SD)] }\end{array}$ & & $692.67(351.28)$ & 0.131 & & $688.42(342.93)$ & 0.954 & \\
\hline $87-490$ & $404(33)$ & $-0.06(1.23)$ & & $332(31)$ & $0.17(0.83)$ & & $0.0001 *$ \\
\hline $491-859$ & $362(30)$ & $0.02(1.24)$ & 0.241 & $419(39)$ & $0.20(1.24)$ & 0.795 & $0.018^{*}$ \\
\hline 860-1543 & $444(37)$ & $0.11(1.40)$ & $\begin{array}{l}0.051 \\
0.373\end{array}$ & $320(30)$ & $0.20(0.95)$ & $\begin{array}{l}0.948 \\
0.797\end{array}$ & 0.092 \\
\hline Systemic diseases & & & 0.546 & & & 0.004 * & \\
\hline Hypertension (\%) & $74(6)$ & $0.15(1.49)$ & & $140(13)$ & $0.30(1.17)$ & & 0.130 \\
\hline Rhinitis, Sinusitis (\%) & $14(1)$ & $-0.01(0.47)$ & 0.986 & $64(6)$ & $0.03(0.82)$ & $\begin{array}{l}0.104 \\
0.083\end{array}$ & 0.824 \\
\hline Hepatitis (\%) & $14(1)$ & $0.32(0.84)$ & $\begin{array}{l}0.201 \\
0.129\end{array}$ & $16(1)$ & $-0.35(0.74)$ & $\begin{array}{l}0.021 \\
0.013 \\
0.145\end{array}$ & $0.022 *$ \\
\hline $\begin{array}{l}\text { Diabetes mellitus, } \\
\text { Hyperlipidemia (\%) }\end{array}$ & $30(2)$ & $-0.15(0.72)$ & $\begin{array}{l}0.504 \\
0.648 \\
0.051\end{array}$ & $68(6)$ & $-0.05(0.84)$ & $\begin{array}{l}0.017 \\
0.007 \\
0.411 \\
0.335\end{array}$ & 0.945 \\
\hline None $(\%)$ & $1078(89)$ & $0.02(1.31)$ & $\begin{array}{c}0.963 \\
0.9050 .132 \\
0.399\end{array}$ & $783(73)$ & $0.22(1.05)$ & 0.619 & 0.00001 * \\
\hline Medicine & & & 0.286 & & & $0.011 *$ & \\
\hline $\begin{array}{c}\text { Aspirin, Hypertension } \\
(\%)\end{array}$ & $30(2)$ & $-0.22(0.98)$ & & $148(14)$ & $0.17(1.02)$ & & 0.107 \\
\hline Anticoagulant (\%) & $32(3)$ & $-0.01(1.44)$ & 0.972 & $0(0)$ & - & - & - \\
\hline $\begin{array}{l}\text { Diabetes mellitus, } \\
\text { hyperlipidemia (\%) }\end{array}$ & $24(2)$ & $0.23(0.51)$ & $\begin{array}{l}0.084 \\
0.071 \\
0.398\end{array}$ & $40(4)$ & $-0.21(0.96)$ & $\begin{array}{c}0.023 \\
0.002\end{array}$ & $0.018^{*}$ \\
\hline None (\%) & $1124(93)$ & $0.03(1.31)$ & $\begin{array}{l}0.391 \\
0.136\end{array}$ & $883(82)$ & $0.21(1.05)$ & 0.621 & 0.00001 * \\
\hline Oral regions & & & 0.568 & & & $0.004 *$ & \\
\hline \#14-17 (\%) & $298(25)$ & $0.04(1.21)$ & & $302(28)$ & $0.32(0.91)$ & & 0.00001 * \\
\hline \#24-27 (\%) & $362(30)$ & $0.10(1.50)$ & 0.893 & $264(25)$ & $0.07(1.05)$ & $0.004 *$ & 0.342 \\
\hline \#34-37 (\%) & $274(23)$ & $-0.03(1.10)$ & $\begin{array}{l}0.379 \\
0.323 \\
0.320\end{array}$ & $232(22)$ & $0.18(0.91)$ & $\begin{array}{c}0.030 \\
0.556 \\
0.001 \text { * }\end{array}$ & 0.024 * \\
\hline \#44-47 (\%) & $276(23)$ & $-0.03(1.29)$ & $\begin{array}{l}0.265 \\
0.880\end{array}$ & $273(25)$ & $0.18(1.25)$ & $\begin{array}{l}0.768 \\
0.427\end{array}$ & 0.087 \\
\hline Precise sites & & & 0.735 & & & 0.537 & \\
\hline \#04 (\%) & $312(26)$ & $-0.03(1.06)$ & & $282(26)$ & $0.11(0.76)$ & & $0.033^{*}$ \\
\hline \#05 (\%) & $312(26)$ & $-0.04(0.94)$ & 0.808 & $282(26)$ & $0.17(0.91)$ & 0.180 & $0.002 *$ \\
\hline \#06 (\%) & $306(25)$ & $0.09(1.23)$ & $\begin{array}{l}0.291 \\
0.380\end{array}$ & $261(24)$ & $0.22(1.04)$ & $\begin{array}{l}0.198 \\
0.979\end{array}$ & 0.061 \\
\hline & & & 0.921 & & & 0.394 & \\
\hline \#07 (\%) & $280(23)$ & $0.10(1.84)$ & 0.961 & $246(23)$ & $0.28(1.40)$ & 0.840 & $0.039 *$ \\
\hline & & & 0.445 & & & 0.830 & \\
\hline
\end{tabular}

$p$ 1: $p$ values among subjects in the GTR or Flap groups $\left({ }^{*}:\right.$ Statistical significance level was $\left.5 \%, p<0.05\right) . p$ 2: $p$ values between the GTR and Flap groups (*: Statistical significance level was $5 \%, p<0.05)$. CBCT interval: Interval between first and second CBCT. \#14-17: Right upper premolar and molar region (FDI numbering system). \#24-27: Left upper premolar and molar region (FDI numbering system). \#34-37: Left lower premolar and molar region (FDI numbering system). \#44-47: Right lower premolar and molar region (FDI numbering system). \#04: First premolars (FDI numbering system). \#05: Second premolars (FDI numbering system). \#06: First molars (FDI numbering system). \#07: Second molars (FDI numbering system). 

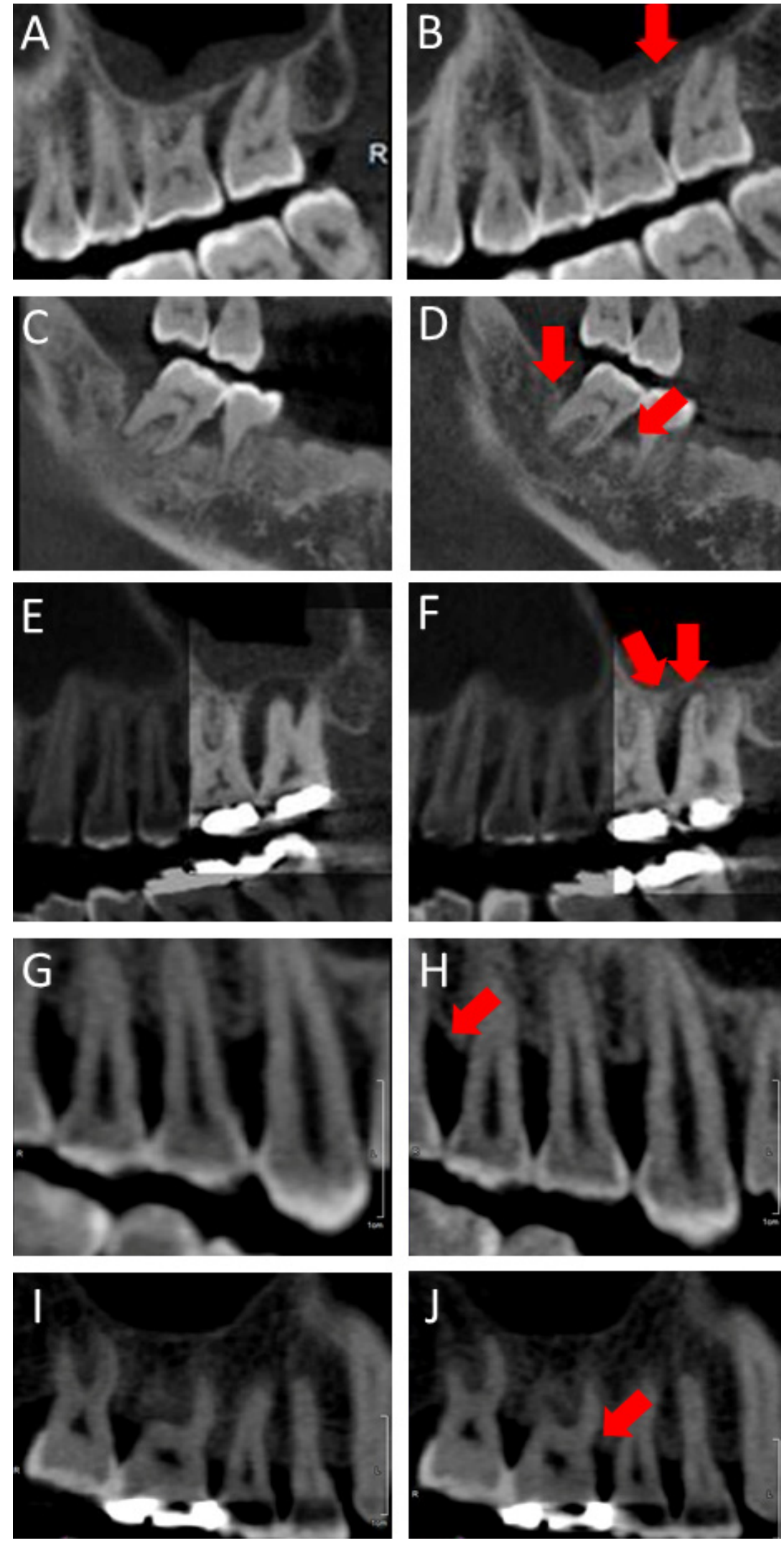

Figure 4. (A-F): $\mathrm{CBCT}$ images before and after treatment in the GTR group. (G-J): $C B C T$ images before and after treatment in the Flap group (Arrow: bone healing area). 


\subsection{Association of Tooth Site and Bone Height between GTR and Flap}

The bony height values of \#14D, \#15M, and \#47M in the GTR group were significantly higher than those in the Flap group $(p=0.016, p=0.036$, and $p=0.023$, for each). In addition, the bony height values of \#14M, \#17D, and \#35M in the GTR group were close to significance ( $p=0.068, p=0.060$, and $p=0.073$, respectively). However, the change of bony height value for \#25D in the Flap group revealed a significant difference to that in the GTR group $(p=0.043)$ (Table 2).

Table 2. Association of tooth site and bone height between GTR and Flap (mm).

\begin{tabular}{|c|c|c|c|c|c|}
\hline \multirow{3}{*}{ Variable } & \multicolumn{5}{|c|}{ Surgery } \\
\hline & \multicolumn{2}{|c|}{ GTR } & \multicolumn{2}{|c|}{ FLAP } & \multirow{2}{*}{$p$} \\
\hline & $N$ & Mean (SD) & $N$ & Mean (SD) & \\
\hline Total & 1210 & $0.03(0.13)$ & 1071 & $0.19(0.10)$ & 0.00001 * \\
\hline \multicolumn{6}{|l|}{ Location } \\
\hline \multicolumn{6}{|l|}{ Site \#10 } \\
\hline$\# 14 \mathrm{M}$ & 39 & $-0.08(1.38)$ & 38 & $0.26(0.67)$ & 0.068 \\
\hline \#14D & 39 & $-0.23(1.21)$ & 38 & $0.32(0.73)$ & $0.016^{*}$ \\
\hline$\# 15 \mathrm{M}$ & 39 & $0.01(0.63)$ & 39 & $0.36(0.64)$ & $0.036^{*}$ \\
\hline$\# 15 D$ & 39 & $0.36(1.04)$ & 39 & $0.30(0.74)$ & 0.730 \\
\hline$\# 16 \mathrm{M}$ & 36 & $0.02(1.18)$ & 38 & $0.21(0.77)$ & 0.584 \\
\hline$\# 16 \mathrm{D}$ & 36 & $0.08(1.39)$ & 38 & 0.32 (1.17) & 0.242 \\
\hline$\# 17 \mathrm{M}$ & 35 & $0.04(0.98)$ & 36 & $0.40(1.04)$ & 0.167 \\
\hline$\# 17 D$ & 35 & $0.13(1.68)$ & 36 & 0.39 (1.34) & 0.060 \\
\hline \multicolumn{6}{|l|}{ Site \#20 } \\
\hline \#24M & 48 & $0.03(0.98)$ & 36 & $-0.05(0.80)$ & 0.978 \\
\hline \#24D & 48 & $-0.09(0.83)$ & 36 & $-0.13(0.87)$ & 0.964 \\
\hline$\# 25 \mathrm{M}$ & 47 & $-0.22(1.19)$ & 34 & $0.14(0.78)$ & 0.244 \\
\hline$\# 25 \mathrm{D}$ & 47 & $-0.29(1.04)$ & 34 & $-0.08(1.33)$ & $0.043 *$ \\
\hline \#26M & 48 & $0.20(1.20)$ & 31 & $0.36(0.97)$ & 0.778 \\
\hline$\# 26 \mathrm{D}$ & 48 & $0.31(1.62)$ & 31 & $0.13(0.88)$ & 0.996 \\
\hline \#27M & 38 & $0.92(2.91)$ & 31 & $0.05(1.58)$ & 0.704 \\
\hline \#27D & 38 & $0.13(1.37)$ & 31 & $0.15(0.97)$ & 0.587 \\
\hline \multicolumn{6}{|l|}{ Site \#30 } \\
\hline \#34M & 33 & $0.05(0.98)$ & 31 & $0.20(0.64)$ & 0.326 \\
\hline \#34D & 33 & $0.04(0.70)$ & 31 & $0.14(0.60)$ & 0.427 \\
\hline \#35M & 35 & $-0.22(0.94)$ & 31 & $0.17(0.68)$ & 0.073 \\
\hline \#35D & 35 & $0.00(0.83)$ & 31 & $0.06(0.79)$ & 0.867 \\
\hline \#36M & 35 & $-0.17(0.84)$ & 27 & $-0.01(0.97)$ & 0.243 \\
\hline \#36D & 35 & $0.01(0.93)$ & 27 & $0.31(0.96)$ & 0.203 \\
\hline \#37M & 34 & $0.22(1.41)$ & 27 & $0.30(1.31)$ & 0.890 \\
\hline \#37D & 34 & $-0.17(1.81)$ & 27 & $0.27(1.23)$ & 0.576 \\
\hline \multicolumn{6}{|l|}{ Site \#40 } \\
\hline \#44M & 36 & $-0.19(1.01)$ & 36 & $0.11(0.98)$ & 0.463 \\
\hline \#44D & 36 & $0.24(1.28)$ & 36 & $-0.01(0.60)$ & 0.426 \\
\hline$\# 45 \mathrm{M}$ & 35 & $0.16(0.84)$ & 37 & $0.08(0.96)$ & 0.660 \\
\hline \#45D & 35 & $-0.02(0.67)$ & 37 & $0.25(1.11)$ & 0.266 \\
\hline$\# 46 \mathrm{M}$ & 34 & $0.21(1.45)$ & 34 & $0.13(1.35)$ & 0.628 \\
\hline$\# 46 \mathrm{D}$ & 34 & $-0.02(0.84)$ & 35 & $0.24(1.19)$ & 0.601 \\
\hline \#47M & 33 & $-0.37(1.28)$ & 29 & $0.47(1.57)$ & 0.023 * \\
\hline \#47D & 33 & $-0.25(2.29)$ & 29 & $0.22(2.08)$ & 0.277 \\
\hline
\end{tabular}

$p: p$ values among subjects in the GTR or Flap groups (*: Statistical significance level was $5 \%, p<0.05)$. GTR: Guided tissue regeneration. \#14-17: Right upper premolar and molar region (FDI numbering system). \#24-27: Left upper premolar and molar region (FDI numbering system). \#34-37: Left lower premolar and molar region (FDI numbering system). \#44-47: Right lower premolar and molar region (FDI numbering system).

\subsection{Association of Sex and Bone Height between GTR and Flap}

In the GTR group, women were more effective at \#14D and \#15M ( $p=0.028$ and $p=0.009$, for each). In males, GTR increased bone resorption at \#15D. In males, the GTR 
group had increased bone resorption at \#15D compared with the Flap group $(0.54 \pm 1.32$; GTR vs. $0.25 \pm 0.85$; Flap, $p=0.635$ ). In the case of $\# 24 \mathrm{D}$, the amount of bony change of GTR group was smaller than that of the Flap group regardless of gender (Male: $0.11 \pm 0.77$; GTR vs. $-0.15 \pm 0.92$; Flap, $p=0.315$, Female: $-0.23 \pm 0.85$; GTR vs. $-0.09 \pm 0.79$; Flap, $p=0.594)$. Both males and females showed better bony healing at \#35 M, but the GTR group was especially effective in females (Male: $-0.18 \pm 1.22$; GTR vs. $0.06 \pm 0.68$; Flap, $p=0.542$, Female: $-0.27 \pm 0.45$; GTR vs. $0.40 \pm 0.66$; Flap, $p=0.012$ ). Comparison of GTR and Flap in males showed significant results at $\# 47 \mathrm{M}(-0.77 \pm 1.32$; GTR vs. $0.39 \pm 1.89$; Flap, $p=0.017$ ) (Table 3$)$.

Table 3. Association of sex and bone height between GTR and Flap (mm).

\begin{tabular}{|c|c|c|c|c|c|c|c|c|c|c|}
\hline \multirow{4}{*}{ Variable } & \multicolumn{10}{|c|}{ Sex } \\
\hline & \multicolumn{5}{|c|}{ Male } & \multicolumn{5}{|c|}{ Female } \\
\hline & \multicolumn{2}{|c|}{ GTR } & \multicolumn{2}{|c|}{ FLAP } & \multirow[b]{2}{*}{$p$} & \multicolumn{2}{|c|}{ GTR } & \multicolumn{2}{|c|}{ FLAP } & \multirow[b]{2}{*}{$p$} \\
\hline & $N$ & $\begin{array}{c}\text { Mean } \\
\text { (SD) }\end{array}$ & $N$ & $\begin{array}{c}\text { Mean } \\
\text { (SD) }\end{array}$ & & $N$ & $\begin{array}{c}\text { Mean } \\
\text { (SD) }\end{array}$ & $N$ & $\begin{array}{l}\text { Mean } \\
\text { (SD) }\end{array}$ & \\
\hline $\begin{array}{c}\text { Total } \\
\text { Location } \\
\text { Site \#10 }\end{array}$ & 594 & $0.00(0.12)$ & 666 & $0.18(0.11)$ & 0.010 * & 616 & $0.05(0.14)$ & 405 & $0.20(0.09)$ & 0.00001 * \\
\hline$\# 14 \mathrm{M}$ & 18 & $\begin{array}{l}-0.16 \\
(1.86)\end{array}$ & 20 & $0.28(0.80)$ & 0.120 & 21 & $\begin{array}{l}-0.01 \\
(0.82)\end{array}$ & 18 & $0.25(0.50)$ & 0.366 \\
\hline$\# 14 \mathrm{D}$ & 18 & $\begin{array}{l}-0.08 \\
(0.74)\end{array}$ & 20 & $0.25(0.71)$ & 0.253 & 21 & $\begin{array}{l}-0.36 \\
(1.51)\end{array}$ & 18 & $0.41(0.76)$ & $0.028 *$ \\
\hline \#15M & 19 & $0.02(0.69)$ & 21 & $0.19(0.62)$ & 0.606 & 20 & $0.01(0.58)$ & 18 & $0.55(0.62)$ & $0.009 *$ \\
\hline$\# 15 \mathrm{D}$ & 19 & $0.54(1.32)$ & 21 & $0.25(0.85)$ & 0.635 & 20 & $0.20(0.67)$ & 18 & $0.36(0.61)$ & 0.285 \\
\hline \#16M & 18 & $0.13(0.62)$ & 20 & $0.19(0.82)$ & 0.769 & 18 & $\begin{array}{l}-0.09 \\
(1.56)\end{array}$ & 18 & $0.23(0.73)$ & 0.635 \\
\hline$\# 16 \mathrm{D}$ & 18 & $\begin{array}{c}-0.03 \\
(0.89)\end{array}$ & 20 & $0.38(1.25)$ & 0.278 & 18 & $0.20(1.77)$ & 18 & $0.27(1.10)$ & 0.569 \\
\hline$\# 17 \mathrm{M}$ & 17 & $0.04(0.69)$ & 18 & $0.53(1.23)$ & 0.215 & 18 & $0.04(1.21)$ & 18 & $0.26(0.82)$ & 0.410 \\
\hline \#17D & 17 & $\begin{array}{c}-0.07 \\
(1.64)\end{array}$ & 18 & $0.44(1.84)$ & 0.203 & 18 & $0.31(1.74)$ & 18 & $0.34(0.58)$ & 0.260 \\
\hline \multicolumn{11}{|l|}{ Site \#20 } \\
\hline \#24M & 20 & $0.27(1.03)$ & 24 & $\begin{array}{c}-0.14 \\
(0.85)\end{array}$ & 0.310 & 28 & $\begin{array}{c}-0.14 \\
(0.92)\end{array}$ & 12 & $0.13(0.68)$ & 0.214 \\
\hline$\# 24 \mathrm{D}$ & 20 & $0.11(0.77)$ & 24 & $\begin{array}{l}-0.15 \\
(0.92)\end{array}$ & 0.315 & 28 & $\begin{array}{l}-0.23 \\
(0.85)\end{array}$ & 12 & $\begin{array}{c}-0.09 \\
(0.79)\end{array}$ & 0.594 \\
\hline$\# 25 \mathrm{M}$ & 20 & $0.07(0.77)$ & 23 & $0.20(0.83)$ & 0.706 & 27 & $\begin{array}{c}-0.43 \\
(1.40)\end{array}$ & 11 & $0.03(0.69)$ & 0.202 \\
\hline$\# 25 \mathrm{D}$ & 20 & $\begin{array}{l}-0.16 \\
(0.66)\end{array}$ & 23 & $\begin{array}{l}-0.21 \\
(1.55)\end{array}$ & 0.227 & 27 & $\begin{array}{l}-0.40 \\
(1.25)\end{array}$ & 11 & $0.20(0.70)$ & 0.084 \\
\hline \#26M & 21 & $0.21(0.76)$ & 19 & $0.28(0.97)$ & 0.734 & 27 & $0.19(1.46)$ & 12 & $0.48(1.00)$ & 0.511 \\
\hline \#26D & 21 & $0.05(0.79)$ & 19 & 0.14 (1.09) & 0.776 & 27 & $0.50(2.05)$ & 12 & $0.12(0.41)$ & 0.681 \\
\hline$\# 27 \mathrm{M}$ & 14 & $0.34(1.14)$ & 20 & $0.02(1.21)$ & 0.612 & 24 & $1.26(3.55)$ & 11 & $0.12(2.18)$ & 0.831 \\
\hline \#27D & 14 & $\begin{array}{l}-0.11 \\
(1.02)\end{array}$ & 20 & $0.19(1.15)$ & 0.889 & 24 & $0.28(1.54)$ & 11 & $0.08(0.56)$ & 0.444 \\
\hline \multicolumn{11}{|l|}{ Site \#30 } \\
\hline \#34M & 18 & $\begin{array}{l}-0.01 \\
(0.93)\end{array}$ & 22 & $0.22(0.70)$ & 0.663 & 15 & $0.11(1.07)$ & 9 & $0.16(0.52)$ & 0.323 \\
\hline \#34D & 18 & $0.17(0.76)$ & 22 & $0.15(0.56)$ & 0.859 & 15 & $\begin{array}{c}-0.11 \\
(0.62)\end{array}$ & 9 & $0.12(0.72)$ & 0.370 \\
\hline \#35M & 19 & $\begin{array}{l}-0.18 \\
(1.22)\end{array}$ & 21 & $0.06(0.68)$ & 0.542 & 16 & $\begin{array}{c}-0.27 \\
(0.45)\end{array}$ & 10 & $0.40(0.66)$ & $0.012 *$ \\
\hline \#35D & 19 & $\begin{array}{l}-0.05 \\
(1.03)\end{array}$ & 21 & $0.18(0.93)$ & 0.480 & 16 & $0.06(0.54)$ & 10 & $\begin{array}{l}-0.19 \\
(0.29)\end{array}$ & 0.169 \\
\hline \#36M & 19 & $\begin{array}{c}-0.18 \\
(1.01)\end{array}$ & 20 & $0.08(0.99)$ & 0.291 & 16 & $\begin{array}{l}-0.15 \\
(0.62)\end{array}$ & 7 & $\begin{array}{l}-0.27 \\
(0.93)\end{array}$ & 0.788 \\
\hline \#36D & 19 & $\begin{array}{l}-0.02 \\
(0.90)\end{array}$ & 20 & $0.46(1.00)$ & 0.109 & 16 & $0.04(0.98)$ & 7 & $\begin{array}{l}-0.11 \\
(0.70)\end{array}$ & 0.867 \\
\hline$\# 37 \mathrm{M}$ & 17 & $0.30(1.51)$ & 19 & $0.35(1.42)$ & 0.886 & 17 & $0.15(1.33)$ & 8 & $0.20(1.10)$ & 0.815 \\
\hline \#37D & 17 & $\begin{array}{l}-0.24 \\
(2.31)\end{array}$ & 19 & $0.28(1.29)$ & 0.775 & 17 & $\begin{array}{l}-0.11 \\
(1.20)\end{array}$ & 8 & $0.23(1.14)$ & 0.640 \\
\hline
\end{tabular}


Table 3. Cont.

\begin{tabular}{|c|c|c|c|c|c|c|c|c|c|c|}
\hline \multirow{4}{*}{ Variable } & \multicolumn{10}{|c|}{ Sex } \\
\hline & \multicolumn{5}{|c|}{ Male } & \multicolumn{5}{|c|}{ Female } \\
\hline & \multicolumn{2}{|c|}{ GTR } & \multicolumn{2}{|c|}{ FLAP } & \multirow[b]{2}{*}{$p$} & \multicolumn{2}{|c|}{ GTR } & \multicolumn{2}{|c|}{ FLAP } & \multirow[b]{2}{*}{$p$} \\
\hline & $N$ & $\begin{array}{c}\text { Mean } \\
\text { (SD) }\end{array}$ & $N$ & $\begin{array}{c}\text { Mean } \\
\text { (SD) }\end{array}$ & & $N$ & $\begin{array}{c}\text { Mean } \\
\text { (SD) }\end{array}$ & $N$ & $\begin{array}{l}\text { Mean } \\
\text { (SD) }\end{array}$ & \\
\hline \multicolumn{11}{|l|}{ Site \#40 } \\
\hline \#44M & 20 & $\begin{array}{l}-0.16 \\
(1.15)\end{array}$ & 24 & $0.20(1.12)$ & 0.351 & 16 & $\begin{array}{l}-0.23 \\
(0.83)\end{array}$ & 12 & $\begin{array}{c}-0.09 \\
(0.62)\end{array}$ & 0.834 \\
\hline \#44D & 20 & $0.26(1.64)$ & 24 & $\begin{array}{l}-0.08 \\
(0.64)\end{array}$ & 0.457 & 16 & $0.21(0.62)$ & 12 & $0.15(0.50)$ & 0.889 \\
\hline$\# 45 \mathrm{M}$ & 19 & $\begin{array}{l}-0.03 \\
(0.79)\end{array}$ & 24 & $\begin{array}{l}-0.03 \\
(1.03)\end{array}$ & 0.990 & 16 & $0.38(0.88)$ & 13 & $0.29(0.79)$ & 0.676 \\
\hline$\# 45 \mathrm{D}$ & 19 & $\begin{array}{l}-0.22 \\
(0.66)\end{array}$ & 24 & $0.30(1.33)$ & 0.126 & 16 & $0.22(0.61)$ & 13 & $0.16(0.54)$ & 0.947 \\
\hline \#46M & 19 & $0.34(1.82)$ & 23 & 0.17 (1.58) & 0.752 & 15 & $0.03(0.80)$ & 11 & $0.05(0.72)$ & 0.658 \\
\hline$\# 46 \mathrm{D}$ & 19 & $0.02(0.69)$ & 23 & $0.22(1.41)$ & 0.889 & 15 & $\begin{array}{l}-0.06 \\
(1.03)\end{array}$ & 12 & $0.28(0.61)$ & 0.282 \\
\hline$\# 47 \mathrm{M}$ & 19 & $\begin{array}{l}-0.77 \\
(1.32)\end{array}$ & 15 & $0.39(1.89)$ & 0.017 * & 14 & $0.19(1.01)$ & 14 & $0.56(1.21)$ & 0.488 \\
\hline \#47D & 19 & $\begin{array}{l}-0.33 \\
(2.85)\end{array}$ & 15 & $0.60(2.45)$ & 0.211 & 14 & $\begin{array}{c}-0.14 \\
(1.28)\end{array}$ & 14 & $\begin{array}{c}-0.19 \\
(1.60)\end{array}$ & 0.782 \\
\hline
\end{tabular}

$p$ : $p$ values among subjects in the GTR or Flap groups ( ${ }^{*}$ : Statistical significance level was $\left.5 \%, p<0.05\right)$. GTR: Guided tissue regeneration. \#14-17: Right upper premolar and molar region (FDI numbering system). \#24-27: Left upper premolar and molar region (FDI numbering system). \#34-37: Left lower premolar and molar region (FDI numbering system). \#44-47: Right lower premolar and molar region (FDI numbering system).

\subsection{Association of Smoking and Bone Height between GTR and Flap}

The GTR and Flap groups differed by smoking status. There was a significant difference between the two groups in non-smoking $(p=0.009)$. Among smokers, 10 of 32 sites in the GTR group showed less bone healing than in the Flap group (\#14M, \#16D, \#24M, \#24D, \#25M, \#27M, \#27D, \#34M, \#44D, and \#46D). In non-smokers, the bone healing of the \#24M and \#34 sites was better in the GTR group ( $p=0.011$ and $p=0.019$, respectively) (Table 4).

Table 4. Association of smoking and bone height between GTR and Flap (mm).

\begin{tabular}{|c|c|c|c|c|c|c|c|c|c|c|c|c|c|c|c|}
\hline \multirow{4}{*}{ Variable } & \multicolumn{15}{|c|}{ Smoking } \\
\hline & \multicolumn{5}{|c|}{ Present } & \multicolumn{5}{|c|}{ Past } & \multicolumn{5}{|c|}{ Not } \\
\hline & \multicolumn{2}{|c|}{ GTR } & \multicolumn{2}{|c|}{ Flap } & \multirow[b]{2}{*}{$p$} & \multicolumn{2}{|c|}{ GTR } & \multicolumn{2}{|c|}{ Flap } & \multirow[b]{2}{*}{$p$} & \multicolumn{2}{|c|}{ GTR } & \multicolumn{2}{|c|}{ Flap } & \multirow[b]{2}{*}{$p$} \\
\hline & $N$ & $\begin{array}{l}\text { Mean } \\
\text { (SD) }\end{array}$ & $N$ & $\begin{array}{c}\text { Mean } \\
\text { (SD) }\end{array}$ & & $N$ & $\begin{array}{c}\text { Mean } \\
\text { (SD) }\end{array}$ & $N$ & $\begin{array}{c}\text { Mean } \\
\text { (SD) }\end{array}$ & & $N$ & $\begin{array}{c}\text { Mean } \\
\text { (SD) }\end{array}$ & $N$ & $\begin{array}{c}\text { Mean } \\
\text { (SD) }\end{array}$ & \\
\hline Total & 280 & $\begin{array}{c}0.10 \\
(0.13)\end{array}$ & 239 & $\begin{array}{c}0.22 \\
(0.12)\end{array}$ & 0.120 & 91 & $\begin{array}{l}-0.05 \\
(0.11)\end{array}$ & 82 & $\begin{array}{c}0.09 \\
(0.11)\end{array}$ & 0.693 & 839 & $\begin{array}{c}0.01 \\
(1.32)\end{array}$ & 750 & $\begin{array}{c}0.19 \\
(0.96)\end{array}$ & $\begin{array}{c}0.009 \\
*\end{array}$ \\
\hline \multicolumn{16}{|l|}{$\begin{array}{l}\text { Location } \\
\text { Site \#10 }\end{array}$} \\
\hline \#14M & 8 & $\begin{array}{c}0.56 \\
(1.87)\end{array}$ & 4 & $\begin{array}{c}0.38 \\
(0.34)\end{array}$ & 0.495 & 3 & $\begin{array}{c}-0.13 \\
(0.12)\end{array}$ & 2 & $\begin{array}{c}0.15 \\
(0.64)\end{array}$ & 1.000 & 28 & $\begin{array}{l}-0.25 \\
(1.27)\end{array}$ & 32 & $\begin{array}{c}0.26 \\
(0.71)\end{array}$ & 0.096 \\
\hline$\# 14 \mathrm{D}$ & 10 & $\begin{array}{l}-0.03 \\
(0.63)\end{array}$ & 9 & $\begin{array}{c}0.33 \\
(0.41)\end{array}$ & 0.205 & 5 & $\begin{array}{c}0.16 \\
(0.45)\end{array}$ & 3 & $\begin{array}{c}0.63 \\
(0.67)\end{array}$ & 0.131 & 24 & $\begin{array}{l}-0.39 \\
(1.46)\end{array}$ & 26 & $\begin{array}{c}0.28 \\
(0.82)\end{array}$ & 0.080 \\
\hline$\# 15 \mathrm{M}$ & 9 & $\begin{array}{l}-0.11 \\
(0.52)\end{array}$ & 6 & $\begin{array}{c}0.13 \\
(0.29)\end{array}$ & 0.473 & 4 & $\begin{array}{l}-0.58 \\
(0.74)\end{array}$ & 1 & $\mathrm{~N} / \mathrm{A}$ & 0.277 & 26 & $\begin{array}{c}0.15 \\
(0.60)\end{array}$ & 32 & $\begin{array}{c}0.40 \\
(0.69)\end{array}$ & 0.194 \\
\hline$\# 15 \mathrm{D}$ & 10 & $\begin{array}{c}0.15 \\
(0.95)\end{array}$ & 11 & $\begin{array}{c}0.47 \\
(1.08)\end{array}$ & 0.501 & 5 & $\begin{array}{c}1.46 \\
(2.07)\end{array}$ & 1 & $\mathrm{~N} / \mathrm{A}$ & 0.546 & 24 & $\begin{array}{c}0.23 \\
(0.62)\end{array}$ & 27 & $\begin{array}{c}0.22 \\
(0.57)\end{array}$ & 0.769 \\
\hline$\# 16 \mathrm{M}$ & 5 & $\begin{array}{l}-0.66 \\
(1.30)\end{array}$ & 5 & $\begin{array}{c}0.02 \\
(1.21)\end{array}$ & 0.465 & 3 & $\begin{array}{c}0.63 \\
(0.50)\end{array}$ & 1 & $\mathrm{~N} / \mathrm{A}$ & 0.180 & 28 & $\begin{array}{c}0.08 \\
(1.18)\end{array}$ & 32 & $\begin{array}{c}0.25 \\
(0.71)\end{array}$ & 0.630 \\
\hline$\# 16 \mathrm{D}$ & 8 & $\begin{array}{c}0.29 \\
(0.38)\end{array}$ & 11 & $\begin{array}{c}0.17 \\
(0.78)\end{array}$ & 0.589 & 6 & $\begin{array}{l}-0.07 \\
(0.58)\end{array}$ & 2 & $\begin{array}{c}0.40 \\
(0.14)\end{array}$ & 0.317 & 22 & $\begin{array}{c}0.05 \\
(1.75)\end{array}$ & 25 & $\begin{array}{c}0.38 \\
(1.35)\end{array}$ & 0.276 \\
\hline \#17M & 3 & $\begin{array}{l}-0.13 \\
(0.95)\end{array}$ & 6 & $\begin{array}{c}0.48 \\
(0.59)\end{array}$ & 0.362 & 1 & $\mathrm{~N} / \mathrm{A}$ & 1 & $\mathrm{~N} / \mathrm{A}$ & 0.317 & 31 & $\begin{array}{c}0.10 \\
(0.98)\end{array}$ & 29 & $\begin{array}{c}0.38 \\
(1.13)\end{array}$ & 0.366 \\
\hline \#17D & 9 & $\begin{array}{c}0.82 \\
(1.92)\end{array}$ & 10 & $\begin{array}{c}0.88 \\
(2.07)\end{array}$ & 0.838 & 3 & $\begin{array}{l}-0.50 \\
(0.52)\end{array}$ & 4 & $\begin{array}{l}-0.23 \\
(0.30)\end{array}$ & 0.368 & 23 & $\begin{array}{l}-0.06 \\
(1.64)\end{array}$ & 22 & $\begin{array}{c}0.28 \\
(0.97)\end{array}$ & 0.044 \\
\hline
\end{tabular}


Table 4. Cont.

\begin{tabular}{|c|c|c|c|c|c|c|c|c|c|c|c|c|c|c|c|}
\hline \multirow{4}{*}{ Variable } & \multicolumn{15}{|c|}{ Smoking } \\
\hline & \multicolumn{5}{|c|}{ Present } & \multicolumn{5}{|c|}{ Past } & \multicolumn{5}{|c|}{ Not } \\
\hline & \multicolumn{2}{|c|}{ GTR } & \multicolumn{2}{|c|}{ Flap } & \multirow[b]{2}{*}{$p$} & \multicolumn{2}{|c|}{ GTR } & \multicolumn{2}{|c|}{ Flap } & \multirow[b]{2}{*}{$p$} & \multicolumn{2}{|c|}{ GTR } & \multicolumn{2}{|c|}{ Flap } & \multirow[b]{2}{*}{$p$} \\
\hline & $N$ & $\begin{array}{c}\text { Mean } \\
\text { (SD) }\end{array}$ & $N$ & $\begin{array}{c}\text { Mean } \\
\text { (SD) }\end{array}$ & & $N$ & $\begin{array}{c}\text { Mean } \\
\text { (SD) }\end{array}$ & $N$ & $\begin{array}{c}\text { Mean } \\
\text { (SD) }\end{array}$ & & $N$ & $\begin{array}{c}\text { Mean } \\
\text { (SD) }\end{array}$ & $N$ & $\begin{array}{c}\text { Mean } \\
\text { (SD) }\end{array}$ & \\
\hline \multicolumn{16}{|l|}{ Site \#20 } \\
\hline \#24M & 9 & $\begin{array}{c}0.69 \\
(1.27)\end{array}$ & 6 & $\begin{array}{c}-0.43 \\
(0.60)\end{array}$ & $0.037^{*}$ & 1 & $\mathrm{~N} / \mathrm{A}$ & 0 & $\mathrm{~N} / \mathrm{A}$ & $\mathrm{N} / \mathrm{A}$ & & & & & \\
\hline$\# 24 \mathrm{D}$ & 12 & $\begin{array}{c}0.36 \\
(0.45)\end{array}$ & 14 & $\begin{array}{l}-0.34 \\
(0.88)\end{array}$ & 0.074 & 5 & $\begin{array}{l}-0.32 \\
(0.99)\end{array}$ & 3 & $\begin{array}{l}-1.03 \\
(1.30)\end{array}$ & 0.294 & 38 & $\begin{array}{l}-0.13 \\
(0.86)\end{array}$ & 30 & $\begin{array}{c}0.02 \\
(0.82)\end{array}$ & 0.332 \\
\hline \#25M & 8 & $\begin{array}{c}0.10 \\
(0.62)\end{array}$ & 7 & $\begin{array}{l}-0.11 \\
(0.89)\end{array}$ & 0.602 & 1 & $\begin{array}{c}\mathrm{N} / \mathrm{A} \\
(\mathrm{N} / \mathrm{A})\end{array}$ & 1 & $\mathrm{~N} / \mathrm{A}$ & 0.317 & 31 & $\begin{array}{l}-0.23 \\
(0.87)\end{array}$ & 19 & $\begin{array}{c}0.17 \\
(0.66)\end{array}$ & 0.126 \\
\hline \#25D & 14 & $\begin{array}{l}-0.09 \\
(0.74)\end{array}$ & 9 & $\begin{array}{c}0.12 \\
(0.69)\end{array}$ & 0.229 & 4 & $\begin{array}{l}-0.83 \\
(0.76)\end{array}$ & 5 & $\begin{array}{c}0.48 \\
(0.53)\end{array}$ & $\begin{array}{c}0.027 \\
*\end{array}$ & 38 & $\begin{array}{l}-0.26 \\
(1.28)\end{array}$ & 26 & $\begin{array}{c}0.25 \\
(0.74)\end{array}$ & 0.089 \\
\hline \#26M & 10 & $\begin{array}{l}-0.26 \\
(0.80)\end{array}$ & 6 & $\begin{array}{c}-0.07 \\
(0.70)\end{array}$ & 0.785 & 2 & $\begin{array}{c}0.15 \\
(0.21)\end{array}$ & 4 & $\begin{array}{c}0.53 \\
(1.36)\end{array}$ & 1.000 & 29 & $\begin{array}{c}-0.32 \\
(1.18)\end{array}$ & 20 & $\begin{array}{l}-0.31 \\
(1.64)\end{array}$ & 0.380 \\
\hline$\# 26 \mathrm{D}$ & 10 & $\begin{array}{c}-0.08 \\
(0.80)\end{array}$ & 9 & $\begin{array}{l}-0.17 \\
(1.17)\end{array}$ & 0.870 & 5 & $\begin{array}{c}0.02 \\
(0.18)\end{array}$ & 3 & $\begin{array}{c}0.80 \\
(0.96)\end{array}$ & 0.453 & 36 & $\begin{array}{c}0.33 \\
(1.29)\end{array}$ & 21 & $\begin{array}{c}0.44 \\
(0.97)\end{array}$ & 0.993 \\
\hline \#27M & 9 & $\begin{array}{c}1.24 \\
(1.49)\end{array}$ & 5 & $\begin{array}{l}-1.06 \\
(1.31)\end{array}$ & $0.019 *$ & 1 & $\mathrm{~N} / \mathrm{A}$ & 3 & $\begin{array}{c}0.67 \\
(0.65)\end{array}$ & 0.180 & 33 & $\begin{array}{c}0.47 \\
(1.90)\end{array}$ & 19 & $\begin{array}{c}0.17 \\
(0.67)\end{array}$ & 0.754 \\
\hline \#27D & 14 & $\begin{array}{c}0.46 \\
(1.48)\end{array}$ & 13 & $\begin{array}{c}0.34 \\
(1.30)\end{array}$ & 0.697 & 1 & $\mathrm{~N} / \mathrm{A}$ & 4 & $\begin{array}{l}-0.03 \\
(0.41)\end{array}$ & 0.717 & 28 & $\begin{array}{c}0.94 \\
(3.24)\end{array}$ & 23 & $\begin{array}{c}0.21 \\
(1.65)\end{array}$ & 0.622 \\
\hline \multicolumn{16}{|l|}{ Site \#30 } \\
\hline \#34M & 6 & $\begin{array}{c}0.10 \\
(0.73)\end{array}$ & 3 & $\begin{array}{c}-0.37 \\
(0.67)\end{array}$ & 0.362 & 2 & $\begin{array}{l}-0.20 \\
(2.12)\end{array}$ & 3 & $\begin{array}{c}-0.07 \\
(0.55)\end{array}$ & 1.000 & 25 & $\begin{array}{c}0.06 \\
(0.99)\end{array}$ & 25 & $\begin{array}{c}0.3 \\
(0.63)\end{array}$ & 0.161 \\
\hline \#34D & 11 & $\begin{array}{c}0.04 \\
(0.65)\end{array}$ & 8 & $\begin{array}{c}0.23 \\
(0.52)\end{array}$ & 0.617 & 2 & $\begin{array}{l}-0.40 \\
(0.28)\end{array}$ & 2 & $\begin{array}{c}-0.35 \\
(0.64)\end{array}$ & 1.000 & 20 & $\begin{array}{c}0.09 \\
(0.76)\end{array}$ & 21 & $\begin{array}{c}0.15 \\
(0.63)\end{array}$ & 0.522 \\
\hline$\# 35 \mathrm{M}$ & 6 & $\begin{array}{l}-1.05 \\
(1.23)\end{array}$ & 2 & $\begin{array}{c}-0.10 \\
(0.14)\end{array}$ & 0.129 & 1 & $\mathrm{~N} / \mathrm{A}$ & 1 & $\mathrm{~N} / \mathrm{A}$ & 0.317 & 28 & $\begin{array}{l}-0.07 \\
(0.80)\end{array}$ & 28 & $\begin{array}{c}0.16 \\
(0.69)\end{array}$ & 0.221 \\
\hline \#35D & 12 & $\begin{array}{l}-0.18 \\
(1.16)\end{array}$ & 10 & $\begin{array}{c}0.36 \\
(0.55)\end{array}$ & 0.289 & 2 & $\begin{array}{c}0.75 \\
(0.07)\end{array}$ & 2 & $\begin{array}{c}0.55 \\
(1.20)\end{array}$ & 1.000 & 21 & $\begin{array}{c}0.03 \\
(0.60)\end{array}$ & 19 & $\begin{array}{l}-0.15 \\
(0.83)\end{array}$ & 0.635 \\
\hline \#36M & 4 & $\begin{array}{l}-0.10 \\
(0.22)\end{array}$ & 4 & $\begin{array}{l}-0.18 \\
(1.98)\end{array}$ & 0.468 & 2 & $\begin{array}{l}-0.50 \\
(0.14)\end{array}$ & 1 & N/A & 0.480 & 29 & $\begin{array}{l}-0.16 \\
(0.92)\end{array}$ & 22 & $\begin{array}{c}0.05 \\
(0.76)\end{array}$ & 0.336 \\
\hline \#36D & 10 & $\begin{array}{l}-0.05 \\
(0.75)\end{array}$ & 9 & $\begin{array}{c}0.37 \\
(0.97)\end{array}$ & 0.487 & 3 & $\begin{array}{c}0.57 \\
(0.90)\end{array}$ & 3 & $\begin{array}{c}0.20 \\
(1.13)\end{array}$ & 0.827 & 22 & $\begin{array}{l}-0.04 \\
(1.01)\end{array}$ & 15 & $\begin{array}{c}0.29 \\
(0.99)\end{array}$ & 0.233 \\
\hline \#37M & 7 & $\begin{array}{c}0.59 \\
(1.51)\end{array}$ & 4 & $\begin{array}{c}1.48 \\
(2.13)\end{array}$ & 0.448 & 3 & $\begin{array}{c}0.50 \\
(0.50)\end{array}$ & 4 & $\begin{array}{c}0.33 \\
(1.30)\end{array}$ & 0.858 & 24 & $\begin{array}{c}0.08 \\
(1.46)\end{array}$ & 19 & $\begin{array}{c}0.05 \\
(1.04)\end{array}$ & 0.961 \\
\hline \#37D & 9 & $\begin{array}{l}-0.51 \\
(2.84)\end{array}$ & 6 & $\begin{array}{c}0.22 \\
(0.93)\end{array}$ & 0.637 & 2 & $\begin{array}{c}0.70 \\
(0.99)\end{array}$ & 4 & $\begin{array}{l}-1.20 \\
(0.37)\end{array}$ & 0.064 & 23 & $\begin{array}{l}-0.12 \\
(1.35)\end{array}$ & 17 & $\begin{array}{c}0.63 \\
(1.21)\end{array}$ & 0.118 \\
\hline \multicolumn{16}{|l|}{ Site \#40 } \\
\hline$\# 44 \mathrm{M}$ & 4 & $\begin{array}{c}0.00 \\
(0.29)\end{array}$ & 8 & $\begin{array}{c}0.59 \\
(1.81)\end{array}$ & 0.495 & 3 & $\begin{array}{l}-0.40 \\
(0.80)\end{array}$ & 2 & $\begin{array}{c}0.10 \\
(0.28)\end{array}$ & 0.564 & 29 & $\begin{array}{l}-0.20 \\
(1.10)\end{array}$ & 26 & $\begin{array}{c}-0.04 \\
(0.57)\end{array}$ & 0.800 \\
\hline$\# 44 \mathrm{D}$ & 10 & $\begin{array}{c}1.12 \\
(1.93)\end{array}$ & 10 & $\begin{array}{l}-0.01 \\
(0.55)\end{array}$ & $0.041 *$ & 3 & $\begin{array}{l}-1.00 \\
(1.28)\end{array}$ & 4 & $\begin{array}{l}-0.60 \\
(1.16)\end{array}$ & 0.724 & 23 & $\begin{array}{c}0.01 \\
(0.55)\end{array}$ & 22 & $\begin{array}{c}0.10 \\
(0.45)\end{array}$ & 0.715 \\
\hline$\# 45 \mathrm{M}$ & 5 & $\begin{array}{l}-0.50 \\
(0.57)\end{array}$ & 5 & $\begin{array}{c}0.84 \\
(1.06)\end{array}$ & $0.028 *$ & 2 & $\begin{array}{c}0.75 \\
(1.06)\end{array}$ & 3 & $\begin{array}{l}-0.03 \\
(1.50)\end{array}$ & 0.564 & 28 & $\begin{array}{c}0.23 \\
(0.83)\end{array}$ & 29 & $\begin{array}{c}-0.04 \\
(0.86)\end{array}$ & 0.143 \\
\hline$\# 45 \mathrm{D}$ & 9 & $\begin{array}{c}-0.03 \\
(0.64)\end{array}$ & 10 & $\begin{array}{c}0.02 \\
(0.77)\end{array}$ & 0.652 & 3 & $\begin{array}{l}-0.17 \\
(1.11)\end{array}$ & 6 & $\begin{array}{c}0.88 \\
(2.27)\end{array}$ & 0.362 & 23 & $\begin{array}{c}0.00 \\
(0.65)\end{array}$ & 21 & $\begin{array}{c}0.18 \\
(0.71)\end{array}$ & 0.409 \\
\hline$\# 46 \mathrm{M}$ & 7 & $\begin{array}{l}-0.03 \\
(0.79)\end{array}$ & 5 & $\begin{array}{l}-0.24 \\
(2.10)\end{array}$ & 0.569 & 1 & $\mathrm{~N} / \mathrm{A}$ & 2 & $\begin{array}{c}0.85 \\
(3.89)\end{array}$ & 1.000 & 26 & $\begin{array}{c}0.24 \\
(1.60)\end{array}$ & 27 & $\begin{array}{c}0.14 \\
(1.00)\end{array}$ & 0.624 \\
\hline \#46D & 15 & $\begin{array}{l}-0.37 \\
(0.93)\end{array}$ & 12 & $\begin{array}{c}0.63 \\
(1.72)\end{array}$ & 0.117 & 3 & $\begin{array}{c}0.20 \\
(0.36)\end{array}$ & 3 & $\begin{array}{c}-0.37 \\
(0.49)\end{array}$ & 0.127 & 16 & $\begin{array}{c}0.27 \\
(0.73)\end{array}$ & 20 & $\begin{array}{c}0.10 \\
(0.78)\end{array}$ & 0.678 \\
\hline$\# 47 \mathrm{M}$ & 6 & $\begin{array}{l}-0.43 \\
(1.01)\end{array}$ & 6 & $\begin{array}{c}0.18 \\
(1.08)\end{array}$ & 0.226 & 4 & $\begin{array}{l}-0.15 \\
(0.34)\end{array}$ & 1 & N/A & 0.480 & 23 & $\begin{array}{l}-0.39 \\
(1.45)\end{array}$ & 22 & $\begin{array}{c}0.59 \\
(1.72)\end{array}$ & 0.058 \\
\hline \#47D & 11 & $\begin{array}{c}0.25 \\
(2.75)\end{array}$ & 6 & $\begin{array}{c}1.40 \\
(3.26)\end{array}$ & 0.392 & 5 & $\begin{array}{l}-0.70 \\
(1.98)\end{array}$ & 3 & $\begin{array}{c}-0.63 \\
(0.60)\end{array}$ & 1.000 & 17 & $\begin{array}{l}-0.44 \\
(2.13)\end{array}$ & 20 & $\begin{array}{c}-0.01 \\
(1.72)\end{array}$ & 0.234 \\
\hline
\end{tabular}

$p: p$ values among subjects in the GTR or Flap groups (*: Statistical significance level was 5\%, $p<0.05)$. GTR: Guided tissue regeneration. \#14-17: Right upper premolar and molar region (FDI numbering system). \#24-27: Left upper premolar and molar region (FDI numbering system). \#34-37: Left lower premolar and molar region (FDI numbering system). \#44-47: Right lower premolar and molar region (FDI numbering system).

\subsection{Association of Age and Bone Height between GTR and Flap}

At 29-45 years of age, 24 sites showed better bone recovery in the GTR group than in the Flap group. Four sites (\#14D, \#35M, \#47M, and \#47D) in the GTR group showed significant differences $(p=0.016, p=0.036, p=0.010$, and $p=0.011$, for each). The 46- 
53 subgroup showed less bone resorption and bone filling in the GTR group than in the Flap group. In the 54-76 subgroup, the number of favorable healing sites in the GTR group was less than in the sites of the other two subgroups. No difference was found in the 54-76 subgroup (Table 5).

Table 5. Association of age and bone height between GTR and Flap (mm).

\begin{tabular}{|c|c|c|c|c|c|c|c|c|c|c|c|c|c|c|c|}
\hline \multirow{4}{*}{ Variable } & \multicolumn{15}{|c|}{ Age } \\
\hline & \multicolumn{5}{|c|}{ 29-45 Years Old } & \multicolumn{5}{|c|}{ 46-53 Years Old } & \multicolumn{5}{|c|}{ 54-76 Years Old } \\
\hline & \multicolumn{2}{|c|}{ GTR } & \multicolumn{2}{|c|}{ FLAP } & \multirow[b]{2}{*}{$p$} & \multicolumn{2}{|c|}{ GTR } & \multicolumn{2}{|c|}{ FLAP } & \multirow[b]{2}{*}{$p$} & \multicolumn{2}{|c|}{ GTR } & \multicolumn{2}{|c|}{ FLAP } & \multirow[b]{2}{*}{$p$} \\
\hline & $N$ & $\begin{array}{c}\text { Mean } \\
\text { (SD) }\end{array}$ & $N$ & $\begin{array}{c}\text { Mean } \\
\text { (SD) }\end{array}$ & & $N$ & $\begin{array}{c}\text { Mean } \\
\text { (SD) }\end{array}$ & $N$ & $\begin{array}{c}\text { Mean } \\
\text { (SD) }\end{array}$ & & $N$ & $\begin{array}{c}\text { Mean } \\
\text { (SD) }\end{array}$ & $N$ & $\begin{array}{l}\text { Mean } \\
\text { (SD) }\end{array}$ & \\
\hline Total & 416 & $\begin{array}{l}-0.17 \\
(0.12)\end{array}$ & 376 & $\begin{array}{c}0.17 \\
(0.10)\end{array}$ & $\begin{array}{c}0.00001 \\
*\end{array}$ & 388 & $\begin{array}{c}0.19 \\
(0.13)\end{array}$ & 312 & $\begin{array}{c}0.24 \\
(0.11)\end{array}$ & 0.040 * & 406 & $\begin{array}{c}0.08 \\
(0.14)\end{array}$ & 383 & $\begin{array}{c}0.16 \\
(0.10)\end{array}$ & 0.125 \\
\hline \multicolumn{16}{|l|}{$\begin{array}{l}\text { Location } \\
\text { Site \#10 }\end{array}$} \\
\hline \#14M & 10 & $\begin{array}{l}-0.23 \\
(0.55)\end{array}$ & 13 & $\begin{array}{c}0.19 \\
(0.39)\end{array}$ & 0.087 & 13 & $\begin{array}{l}-0.02 \\
(0.46)\end{array}$ & 10 & $\begin{array}{c}0.12 \\
(0.78)\end{array}$ & 0.618 & 16 & $\begin{array}{l}-0.03 \\
(2.11)\end{array}$ & 15 & $\begin{array}{c}0.43 \\
(0.78)\end{array}$ & 0.352 \\
\hline$\# 14 \mathrm{D}$ & 10 & $\begin{array}{l}-0.32 \\
(0.66)\end{array}$ & 13 & $\begin{array}{c}0.26 \\
(0.41)\end{array}$ & 0.016 * & 13 & $\begin{array}{c}0.03 \\
(0.55)\end{array}$ & 10 & $\begin{array}{c}0.43 \\
(1.06)\end{array}$ & 0.223 & 16 & $\begin{array}{l}-0.38 \\
(1.76)\end{array}$ & 15 & $\begin{array}{c}0.31 \\
(0.72)\end{array}$ & 0.332 \\
\hline$\# 15 \mathrm{M}$ & 11 & $\begin{array}{l}-0.03 \\
(0.56)\end{array}$ & 13 & $\begin{array}{c}0.22 \\
(0.51)\end{array}$ & 0.337 & 12 & $\begin{array}{l}-0.01 \\
(0.72)\end{array}$ & 10 & $\begin{array}{c}0.34 \\
(0.52)\end{array}$ & 0.186 & 16 & $\begin{array}{c}0.06 \\
(0.63)\end{array}$ & 16 & $\begin{array}{c}0.48 \\
(0.80)\end{array}$ & 0.162 \\
\hline \#15D & 11 & $\begin{array}{c}0.66 \\
(1.71)\end{array}$ & 13 & $\begin{array}{c}0.19 \\
(0.67)\end{array}$ & 0.642 & 12 & $\begin{array}{c}0.18 \\
(0.56)\end{array}$ & 10 & $\begin{array}{c}0.34 \\
(0.62)\end{array}$ & 0.427 & 16 & $\begin{array}{c}0.30 \\
(0.67)\end{array}$ & 16 & $\begin{array}{c}0.36 \\
(0.88)\end{array}$ & 0.777 \\
\hline$\# 16 \mathrm{M}$ & 9 & $\begin{array}{l}-0.71 \\
(1.10)\end{array}$ & 13 & $\begin{array}{c}0.14 \\
(0.67)\end{array}$ & 0.076 & 12 & $\begin{array}{c}0.48 \\
(0.77)\end{array}$ & 9 & $\begin{array}{c}0.30 \\
(1.03)\end{array}$ & 0.943 & 15 & $\begin{array}{c}0.09 \\
(1.33)\end{array}$ & 16 & $\begin{array}{c}0.21 \\
(0.73)\end{array}$ & 0.905 \\
\hline$\# 16 \mathrm{D}$ & 9 & $\begin{array}{l}-0.32 \\
(1.24)\end{array}$ & 13 & $\begin{array}{c}0.20 \\
(0.92)\end{array}$ & 0.192 & 12 & $\begin{array}{c}0.06 \\
(0.76)\end{array}$ & 9 & $\begin{array}{c}0.78 \\
(1.53)\end{array}$ & 0.251 & 15 & $\begin{array}{c}0.35 \\
(1.82)\end{array}$ & 16 & $\begin{array}{c}0.17 \\
(1.13)\end{array}$ & 0.782 \\
\hline$\# 17 \mathrm{M}$ & 8 & $\begin{array}{l}-0.58 \\
(1.30)\end{array}$ & 13 & $\begin{array}{c}0.39 \\
(1.37)\end{array}$ & 0.180 & 12 & $\begin{array}{c}0.1 \\
(0.63)\end{array}$ & 7 & $\begin{array}{c}0.63 \\
(0.51)\end{array}$ & 0.074 & 15 & $\begin{array}{c}0.32 \\
(0.92)\end{array}$ & 16 & $\begin{array}{c}0.30 \\
(0.94)\end{array}$ & 0.766 \\
\hline \#17D & 8 & $\begin{array}{l}-0.43 \\
(1.02)\end{array}$ & 13 & $\begin{array}{c}0.35 \\
(1.83)\end{array}$ & 0.514 & 12 & $\begin{array}{c}0.67 \\
(2.44)\end{array}$ & 7 & $\begin{array}{c}0.06 \\
(0.57)\end{array}$ & 1.000 & 15 & $\begin{array}{l}-0.01 \\
(1.10)\end{array}$ & 16 & $\begin{array}{c}0.56 \\
(1.14)\end{array}$ & 0.038 \\
\hline \multicolumn{16}{|l|}{ Site \#20 } \\
\hline \#24M & 16 & $\begin{array}{c}0.03 \\
(1.04)\end{array}$ & 14 & $\begin{array}{c}-0.05 \\
(0.76)\end{array}$ & 0.532 & 17 & $\begin{array}{c}0.03 \\
(0.76)\end{array}$ & 11 & $\begin{array}{c}0.02 \\
(1.08)\end{array}$ & 0.981 & 15 & $\begin{array}{c}0.03 \\
(1.19)\end{array}$ & 11 & $\begin{array}{l}-0.13 \\
(0.57)\end{array}$ & 0.549 \\
\hline \#24D & 16 & $\begin{array}{c}0.13 \\
(0.53)\end{array}$ & 14 & $\begin{array}{l}-0.11 \\
(0.67)\end{array}$ & 0.631 & 17 & $\begin{array}{c}0.08 \\
(0.73)\end{array}$ & 11 & $\begin{array}{c}0.36 \\
(0.87)\end{array}$ & 0.239 & 15 & $\begin{array}{l}-0.52 \\
(1.05)\end{array}$ & 11 & $\begin{array}{l}-0.64 \\
(0.87)\end{array}$ & 0.585 \\
\hline \#25M & 15 & $\begin{array}{l}-0.29 \\
(1.76)\end{array}$ & 14 & $\begin{array}{l}0.07 \\
(0.6)\end{array}$ & 0.930 & 17 & $\begin{array}{l}-0.08 \\
(0.55)\end{array}$ & 10 & $\begin{array}{c}0.40 \\
(0.98)\end{array}$ & 0.174 & 15 & $\begin{array}{c}-0.3 \\
(1.08)\end{array}$ & 10 & $\begin{array}{l}-0.01 \\
(0.81)\end{array}$ & 0.469 \\
\hline$\# 25 \mathrm{D}$ & 15 & $\begin{array}{l}-0.39 \\
(1.47)\end{array}$ & 14 & $\begin{array}{c}-0.08 \\
(0.93)\end{array}$ & 0.457 & 17 & $\begin{array}{c}-0.13 \\
(0.73)\end{array}$ & 10 & $\begin{array}{l}-0.11 \\
(2.23)\end{array}$ & $0.050 *$ & 15 & $\begin{array}{l}-0.39 \\
(0.85)\end{array}$ & 10 & $\begin{array}{l}-0.04 \\
(0.56)\end{array}$ & 0.502 \\
\hline$\# 26 \mathrm{M}$ & 16 & $\begin{array}{l}-0.21 \\
(0.94)\end{array}$ & 10 & $\begin{array}{c}0.08 \\
(0.58)\end{array}$ & 0.544 & 17 & $\begin{array}{c}0.17 \\
(0.74)\end{array}$ & 9 & $\begin{array}{c}0.39 \\
(1.02)\end{array}$ & 0.808 & 15 & $\begin{array}{c}0.67 \\
(1.67)\end{array}$ & 12 & $\begin{array}{c}0.56 \\
(1.19)\end{array}$ & 0.825 \\
\hline \#26D & 16 & $\begin{array}{c}-0.35 \\
(1.14)\end{array}$ & 10 & $\begin{array}{c}0.11 \\
(0.81)\end{array}$ & 0.398 & 17 & $\begin{array}{c}0.29 \\
(0.79)\end{array}$ & 9 & $\begin{array}{c}0.34 \\
(0.83)\end{array}$ & 0.646 & 15 & $\begin{array}{c}1.02 \\
(2.39)\end{array}$ & 12 & $\begin{array}{l}-0.01 \\
(1.01)\end{array}$ & 0.170 \\
\hline \#27M & 11 & $\begin{array}{l}-0.57 \\
(1.39)\end{array}$ & 12 & $\begin{array}{c}0.03 \\
(1.08)\end{array}$ & 0.281 & 14 & $\begin{array}{c}1.84 \\
(3.48)\end{array}$ & 9 & $\begin{array}{c}0.61 \\
(1.34)\end{array}$ & 0.614 & 13 & $\begin{array}{c}1.20 \\
(2.89)\end{array}$ & 10 & $\begin{array}{l}-0.43 \\
(2.18)\end{array}$ & 0.238 \\
\hline \#27D & 11 & $\begin{array}{l}-0.32 \\
(1.05)\end{array}$ & 12 & $\begin{array}{l}-0.11 \\
(1.39)\end{array}$ & 0.711 & 14 & $\begin{array}{c}0.06 \\
(1.26)\end{array}$ & 9 & $\begin{array}{c}0.60 \\
(0.50)\end{array}$ & 0.072 & 13 & $\begin{array}{c}0.60 \\
(1.64)\end{array}$ & 10 & $\begin{array}{c}0.05 \\
(0.50)\end{array}$ & 0.071 \\
\hline \multicolumn{16}{|l|}{ Site \#30 } \\
\hline \#34M & 16 & $\begin{array}{l}-0.07 \\
(0.67)\end{array}$ & 11 & $\begin{array}{c}0.16 \\
(0.49)\end{array}$ & 0.308 & 10 & $\begin{array}{c}0.49 \\
(1.17)\end{array}$ & 9 & $\begin{array}{c}-0.03 \\
(0.77)\end{array}$ & 0.436 & 7 & $\begin{array}{c}-0.31 \\
(1.19)\end{array}$ & 11 & $\begin{array}{c}0.43 \\
(0.65)\end{array}$ & 0.220 \\
\hline \#34D & 16 & $\begin{array}{c}0.05 \\
(0.66)\end{array}$ & 11 & $\begin{array}{c}0.06 \\
(0.52)\end{array}$ & 0.921 & 10 & $\begin{array}{c}0.09 \\
(0.53)\end{array}$ & 9 & $\begin{array}{c}0.43 \\
(0.55)\end{array}$ & 0.175 & 7 & $\begin{array}{l}-0.04 \\
(1.05)\end{array}$ & 11 & $\begin{array}{l}-0.03 \\
(0.67)\end{array}$ & 0.555 \\
\hline \#35M & 17 & $\begin{array}{l}-0.29 \\
(0.68)\end{array}$ & 11 & $\begin{array}{c}0.36 \\
(0.78)\end{array}$ & 0.036 * & 11 & $\begin{array}{l}-0.20 \\
(0.53)\end{array}$ & 9 & $\begin{array}{l}-0.04 \\
(0.65)\end{array}$ & 0.760 & 7 & $\begin{array}{l}-0.09 \\
(1.80)\end{array}$ & 11 & $\begin{array}{l}0.16 \\
(0.6)\end{array}$ & 0.496 \\
\hline \#35D & 17 & $\begin{array}{l}-0.03 \\
(0.71)\end{array}$ & 11 & $\begin{array}{c}0.36 \\
(0.58)\end{array}$ & 0.229 & 11 & $\begin{array}{l}0.15 \\
(0.6)\end{array}$ & 9 & $\begin{array}{l}-0.27 \\
(0.82)\end{array}$ & 0.237 & 7 & $\begin{array}{l}-0.16 \\
(1.38)\end{array}$ & 11 & $\begin{array}{c}0.04 \\
(0.91)\end{array}$ & 0.964 \\
\hline \#36M & 17 & $\begin{array}{l}-0.26 \\
(0.62)\end{array}$ & 9 & $\begin{array}{c}-0.4 \\
(1.36)\end{array}$ & 0.552 & 11 & $\begin{array}{c}0.00 \\
(0.67)\end{array}$ & 8 & $\begin{array}{c}0.19 \\
(0.68)\end{array}$ & 0.455 & 7 & $\begin{array}{l}-0.21 \\
(1.46)\end{array}$ & 10 & $\begin{array}{c}0.18 \\
(0.70)\end{array}$ & 0.961 \\
\hline \#36D & 17 & $\begin{array}{l}-0.08 \\
(0.83)\end{array}$ & 9 & $\begin{array}{c}0.36 \\
(0.58)\end{array}$ & 0.124 & 11 & $\begin{array}{l}-0.04 \\
(1.02)\end{array}$ & 8 & $\begin{array}{c}0.29 \\
(1.11)\end{array}$ & 0.508 & 7 & $\begin{array}{c}0.30 \\
(1.08)\end{array}$ & 10 & $\begin{array}{c}0.28 \\
(1.17)\end{array}$ & 0.660 \\
\hline \#37M & 18 & $\begin{array}{c}0.02 \\
(1.58)\end{array}$ & 9 & $\begin{array}{l}-0.07 \\
(1.25)\end{array}$ & 0.837 & 9 & $\begin{array}{c}0.50 \\
(1.14)\end{array}$ & 7 & $\begin{array}{c}-0.37 \\
(0.83)\end{array}$ & 0.090 & 7 & $\begin{array}{c}0.40 \\
(1.32)\end{array}$ & 11 & $\begin{array}{l}1.04 \\
(1.34)\end{array}$ & 0.585 \\
\hline \#37D & 18 & $\begin{array}{l}-0.24 \\
(2.14)\end{array}$ & 9 & $\begin{array}{c}0.36 \\
(1.21)\end{array}$ & 0.381 & 9 & $\begin{array}{c}0.2 \\
(1.55)\end{array}$ & 7 & $\begin{array}{c}-0.63 \\
(0.57)\end{array}$ & 0.080 & 7 & $\begin{array}{l}-0.49 \\
(1.22)\end{array}$ & 11 & $\begin{array}{c}0.76 \\
(1.31)\end{array}$ & 0.146 \\
\hline
\end{tabular}


Table 5. Cont.

\begin{tabular}{|c|c|c|c|c|c|c|c|c|c|c|c|c|c|c|c|}
\hline \multirow{4}{*}{ Variable } & \multicolumn{15}{|c|}{ Age } \\
\hline & \multicolumn{5}{|c|}{ 29-45 Years Old } & \multicolumn{5}{|c|}{ 46-53 Years Old } & \multicolumn{5}{|c|}{ 54-76 Years Old } \\
\hline & \multicolumn{2}{|c|}{ GTR } & \multicolumn{2}{|c|}{ FLAP } & \multirow[b]{2}{*}{$p$} & \multicolumn{2}{|c|}{ GTR } & \multicolumn{2}{|c|}{ FLAP } & \multirow[b]{2}{*}{$p$} & \multicolumn{2}{|c|}{ GTR } & \multicolumn{2}{|c|}{ FLAP } & \multirow[b]{2}{*}{$p$} \\
\hline & $N$ & $\begin{array}{c}\text { Mean } \\
\text { (SD) }\end{array}$ & $N$ & $\begin{array}{c}\text { Mean } \\
\text { (SD) }\end{array}$ & & $N$ & $\begin{array}{c}\text { Mean } \\
\text { (SD) }\end{array}$ & $N$ & $\begin{array}{c}\text { Mean } \\
\text { (SD) }\end{array}$ & & $N$ & $\begin{array}{c}\text { Mean } \\
\text { (SD) }\end{array}$ & $N$ & $\begin{array}{l}\text { Mean } \\
\text { (SD) }\end{array}$ & \\
\hline \multicolumn{16}{|l|}{ Site \#40 } \\
\hline \#44M & 12 & $\begin{array}{c}-0.14 \\
(1.19)\end{array}$ & 12 & $\begin{array}{c}0.25 \\
(0.55)\end{array}$ & 0.469 & 10 & $\begin{array}{c}0.01 \\
(1.03)\end{array}$ & 13 & $\begin{array}{c}0.32 \\
(1.47)\end{array}$ & 0.755 & 14 & $\begin{array}{c}-0.38 \\
(0.85)\end{array}$ & 11 & $\begin{array}{l}-0.31 \\
(0.39)\end{array}$ & 0.600 \\
\hline \#44D & 12 & $\begin{array}{c}0.37 \\
(2.06)\end{array}$ & 12 & $\begin{array}{c}0.23 \\
(0.47)\end{array}$ & 0.469 & 10 & $\begin{array}{c}0.10 \\
(0.35)\end{array}$ & 13 & $\begin{array}{c}0.06 \\
(0.72)\end{array}$ & 0.619 & 14 & $\begin{array}{c}0.22 \\
(0.83)\end{array}$ & 11 & $\begin{array}{l}-0.35 \\
(0.45)\end{array}$ & 0.061 \\
\hline$\# 45 \mathrm{M}$ & 11 & $\begin{array}{c}0.33 \\
(0.75)\end{array}$ & 12 & $\begin{array}{c}0.24 \\
(0.83)\end{array}$ & 0.643 & 10 & $\begin{array}{c}0.25 \\
(0.74)\end{array}$ & 13 & $\begin{array}{c}0.22 \\
(0.96)\end{array}$ & 0.828 & 14 & $\begin{array}{l}-0.04 \\
(0.99)\end{array}$ & 12 & $\begin{array}{l}-0.23 \\
(1.07)\end{array}$ & 0.680 \\
\hline$\# 45 \mathrm{D}$ & 11 & $\begin{array}{c}0.12 \\
(0.57)\end{array}$ & 12 & $\begin{array}{c}0.38 \\
(0.36)\end{array}$ & 0.153 & 10 & $\begin{array}{c}-0.18 \\
(0.74)\end{array}$ & 13 & $\begin{array}{c}0.58 \\
(1.71)\end{array}$ & 0.144 & 14 & $\begin{array}{c}-0.01 \\
(0.70)\end{array}$ & 12 & $\begin{array}{l}-0.23 \\
(0.56)\end{array}$ & 0.353 \\
\hline$\# 46 \mathrm{M}$ & 11 & $\begin{array}{c}0.24 \\
(0.72)\end{array}$ & 12 & $\begin{array}{l}-0.02 \\
(1.13)\end{array}$ & 0.478 & 10 & $\begin{array}{c}0.36 \\
(2.17)\end{array}$ & 13 & $\begin{array}{c}0.37 \\
(1.75)\end{array}$ & 0.153 & 13 & $\begin{array}{c}0.06 \\
(1.33)\end{array}$ & 9 & $\begin{array}{l}-0.03 \\
(1.03)\end{array}$ & 0.947 \\
\hline$\# 46 \mathrm{D}$ & 11 & $\begin{array}{c}0.19 \\
(0.96)\end{array}$ & 12 & $\begin{array}{c}0.13 \\
(1.39)\end{array}$ & 0.579 & 10 & $\begin{array}{c}0.15 \\
(0.60)\end{array}$ & 13 & $\begin{array}{c}0.39 \\
(1.34)\end{array}$ & 0.733 & 13 & $\begin{array}{l}-0.32 \\
(0.87)\end{array}$ & 10 & $\begin{array}{c}0.19 \\
(0.71)\end{array}$ & 0.250 \\
\hline$\# 47 \mathrm{M}$ & 10 & $\begin{array}{l}-1.05 \\
(1.64)\end{array}$ & 10 & $\begin{array}{c}0.69 \\
(2.12)\end{array}$ & 0.010 * & 9 & $\begin{array}{l}-0.10 \\
(1.15)\end{array}$ & 9 & $\begin{array}{l}-0.09 \\
(1.16)\end{array}$ & 0.965 & 14 & $\begin{array}{l}-0.05 \\
(0.89)\end{array}$ & 10 & $\begin{array}{c}0.76 \\
(1.25)\end{array}$ & 0.150 \\
\hline \#47D & 10 & $\begin{array}{l}-1.64 \\
(2.05)\end{array}$ & 10 & $\begin{array}{c}0.77 \\
(2.64)\end{array}$ & $0.011 *$ & 9 & $\begin{array}{c}0.88 \\
(3.29)\end{array}$ & 9 & $\begin{array}{c}0.28 \\
(1.61)\end{array}$ & 0.626 & 14 & $\begin{array}{c}0.02 \\
(0.95)\end{array}$ & 10 & $\begin{array}{l}-0.39 \\
(1.86)\end{array}$ & 0.953 \\
\hline
\end{tabular}

$p: p$ values among subjects in the GTR or Flap groups $(*$ : Statistical significance level was $5 \%, p<0.05)$. GTR: Guided tissue regeneration. \#14-17: Right upper premolar and molar region (FDI numbering system). \#24-27: Left upper premolar and molar region (FDI numbering system). \#34-37: Left lower premolar and molar region (FDI numbering system). \#44-47: Right lower premolar and molar region (FDI numbering system).

\subsection{Association of CBCT Interval and Bone Height between GTR and Flap}

The smaller the interval between before and after CBCT, the more bone increase and the less bone resorption. In particular, in the 860-1543 days group, there was a significant difference in bone growth or small bone resorption at \#14D, \#26M, and \#35M ( $p=0.048$, $p=0.032$, and $p=0.042$, respectively) (Table 6).

Table 6. Association of CBCT interval and bone height between GTR and Flap (mm).

\begin{tabular}{|c|c|c|c|c|c|c|c|c|c|c|c|c|c|c|c|}
\hline \multirow{4}{*}{ Variable } & \multicolumn{15}{|c|}{ CBCT Interval } \\
\hline & \multicolumn{5}{|c|}{ 87-490 Days } & \multicolumn{5}{|c|}{ 491-859 Days } & \multicolumn{5}{|c|}{ 860-1543 Days } \\
\hline & \multicolumn{2}{|c|}{ GTR } & \multicolumn{2}{|c|}{ Flap } & \multirow[b]{2}{*}{$p$} & \multicolumn{2}{|c|}{ GTR } & \multicolumn{2}{|c|}{ Flap } & \multirow[b]{2}{*}{$p$} & \multicolumn{2}{|c|}{ GTR } & \multicolumn{2}{|c|}{ Flap } & \multirow[b]{2}{*}{$p$} \\
\hline & $N$ & $\begin{array}{c}\text { Mean } \\
\text { (SD) }\end{array}$ & $N$ & $\begin{array}{c}\text { Mean } \\
\text { (SD) }\end{array}$ & & $N$ & $\begin{array}{c}\text { Mean } \\
\text { (SD) }\end{array}$ & $N$ & $\begin{array}{c}\text { Mean } \\
\text { (SD) }\end{array}$ & & $N$ & $\begin{array}{c}\text { Mean } \\
\text { (SD) }\end{array}$ & $N$ & $\begin{array}{c}\text { Mean } \\
\text { (SD) }\end{array}$ & \\
\hline Total & 404 & $\begin{array}{c}-0.06 \\
(0.12)\end{array}$ & 332 & $\begin{array}{c}0.17 \\
(0.08)\end{array}$ & $\begin{array}{c}0.00001 \\
*\end{array}$ & 362 & $\begin{array}{c}0.02 \\
(0.12)\end{array}$ & 419 & $\begin{array}{c}0.20 \\
(0.12)\end{array}$ & 0.018 * & 444 & $\begin{array}{c}0.11 \\
(0.14)\end{array}$ & 320 & $\begin{array}{c}0.20 \\
(0.10)\end{array}$ & 0.092 \\
\hline \multicolumn{16}{|l|}{$\begin{array}{c}\text { Location } \\
\text { Site \#10 }\end{array}$} \\
\hline \#14M & 11 & $\begin{array}{l}-0.30 \\
(0.91)\end{array}$ & 14 & $\begin{array}{c}0.20 \\
(0.51)\end{array}$ & 0.084 & 12 & $\begin{array}{c}0.10 \\
(0.56)\end{array}$ & 18 & $\begin{array}{c}0.33 \\
(0.85)\end{array}$ & 0.308 & 16 & $\begin{array}{l}-0.06 \\
(2.00)\end{array}$ & 6 & $\begin{array}{c}0.20 \\
(0.34)\end{array}$ & 0.529 \\
\hline$\# 14 \mathrm{D}$ & 11 & $\begin{array}{l}-0.06 \\
(0.70)\end{array}$ & 14 & $\begin{array}{c}0.54 \\
(0.85)\end{array}$ & 0.094 & 12 & $\begin{array}{l}-0.73 \\
(1.94)\end{array}$ & 18 & $\begin{array}{c}0.26 \\
(0.67)\end{array}$ & $0.048 *$ & 16 & $\begin{array}{c}0.04 \\
(0.56)\end{array}$ & 6 & $\begin{array}{c}0.02 \\
(0.52)\end{array}$ & 0.912 \\
\hline$\# 15 \mathrm{M}$ & 11 & $\begin{array}{c}-0.14 \\
(0.62)\end{array}$ & 14 & $\begin{array}{c}0.26 \\
(0.63)\end{array}$ & 0.146 & 12 & $\begin{array}{l}-0.05 \\
(0.71)\end{array}$ & 19 & $\begin{array}{c}0.46 \\
(0.71)\end{array}$ & 0.084 & 16 & $\begin{array}{c}0.16 \\
(0.58)\end{array}$ & 6 & $\begin{array}{c}0.23 \\
(0.45)\end{array}$ & 0.767 \\
\hline$\# 15 \mathrm{D}$ & 11 & $\begin{array}{l}-0.06 \\
(0.63)\end{array}$ & 14 & $\begin{array}{c}0.33 \\
(0.76)\end{array}$ & 0.138 & 12 & $\begin{array}{c}0.54 \\
(0.78)\end{array}$ & 19 & $\begin{array}{c}0.31 \\
(0.82)\end{array}$ & 0.502 & 16 & $\begin{array}{c}0.53 \\
(1.35)\end{array}$ & 6 & $\begin{array}{c}0.20 \\
(0.46)\end{array}$ & 0.795 \\
\hline \#16M & 11 & $\begin{array}{l}-0.11 \\
(1.29)\end{array}$ & 14 & $\begin{array}{c}0.38 \\
(0.74)\end{array}$ & 0.285 & 11 & $\begin{array}{c}0.23 \\
(1.32)\end{array}$ & 17 & $\begin{array}{c}0.23 \\
(0.77)\end{array}$ & 0.869 & 14 & $\begin{array}{c}-0.04 \\
(1.02)\end{array}$ & 7 & $\begin{array}{l}-0.19 \\
(0.79)\end{array}$ & 0.525 \\
\hline$\# 16 \mathrm{D}$ & 11 & $\begin{array}{l}-0.44 \\
(1.23)\end{array}$ & 14 & $\begin{array}{c}0.14 \\
(0.74)\end{array}$ & 0.188 & 11 & $\begin{array}{c}0.50 \\
(2.05)\end{array}$ & 17 & $\begin{array}{c}0.64 \\
(1.54)\end{array}$ & 0.247 & 14 & $\begin{array}{c}0.16 \\
(0.65)\end{array}$ & 7 & $\begin{array}{l}-0.07 \\
(0.63)\end{array}$ & 0.501 \\
\hline$\# 17 \mathrm{M}$ & 9 & $\begin{array}{c}-0.26 \\
(1.12)\end{array}$ & 12 & $\begin{array}{c}0.57 \\
(0.71)\end{array}$ & $0.039 *$ & 11 & $\begin{array}{c}0.23 \\
(0.81)\end{array}$ & 18 & $\begin{array}{c}0.42 \\
(1.17)\end{array}$ & 0.770 & 15 & $\begin{array}{c}0.08 \\
(1.02)\end{array}$ & 6 & $\begin{array}{c}0.00 \\
(1.24)\end{array}$ & 0.969 \\
\hline \#17D & 9 & $\begin{array}{l}-0.01 \\
(0.98)\end{array}$ & 12 & $\begin{array}{c}0.38 \\
(1.02)\end{array}$ & 0.545 & 11 & $\begin{array}{c}0.50 \\
(2.18)\end{array}$ & 18 & $\begin{array}{c}0.32 \\
(1.73)\end{array}$ & 0.787 & 15 & $\begin{array}{l}-0.06 \\
(1.65)\end{array}$ & 6 & $\begin{array}{c}0.60 \\
(0.36)\end{array}$ & $0.017^{*}$ \\
\hline
\end{tabular}


Table 6. Cont.

\begin{tabular}{|c|c|c|c|c|c|c|c|c|c|c|c|c|c|c|c|}
\hline \multirow{4}{*}{ Variable } & \multicolumn{15}{|c|}{ CBCT Interval } \\
\hline & \multicolumn{5}{|c|}{ 87-490 Days } & \multicolumn{5}{|c|}{ 491-859 Days } & \multicolumn{5}{|c|}{ 860-1543 Days } \\
\hline & \multicolumn{2}{|c|}{ GTR } & \multicolumn{2}{|c|}{ Flap } & \multirow[b]{2}{*}{$p$} & \multicolumn{2}{|c|}{ GTR } & \multicolumn{2}{|c|}{ Flap } & \multirow[b]{2}{*}{$p$} & \multicolumn{2}{|c|}{ GTR } & \multicolumn{2}{|c|}{ Flap } & \multirow[b]{2}{*}{$p$} \\
\hline & $N$ & $\begin{array}{c}\text { Mean } \\
\text { (SD) }\end{array}$ & $N$ & $\begin{array}{c}\text { Mean } \\
\text { (SD) }\end{array}$ & & $N$ & $\begin{array}{c}\text { Mean } \\
\text { (SD) }\end{array}$ & $N$ & $\begin{array}{c}\text { Mean } \\
\text { (SD) }\end{array}$ & & $N$ & $\begin{array}{c}\text { Mean } \\
\text { (SD) }\end{array}$ & $N$ & $\begin{array}{c}\text { Mean } \\
\text { (SD) }\end{array}$ & \\
\hline \multicolumn{16}{|l|}{ Site \#20 } \\
\hline \#24M & 18 & $\begin{array}{l}-0.19 \\
(0.50)\end{array}$ & 13 & $\begin{array}{c}0.10 \\
(0.69)\end{array}$ & 0.074 & 12 & $\begin{array}{c}0.13 \\
(0.93)\end{array}$ & 11 & $\begin{array}{c}0.14 \\
(0.95)\end{array}$ & 0.734 & 18 & $\begin{array}{c}0.18 \\
(1.33)\end{array}$ & 12 & $\begin{array}{c}-0.39 \\
(0.71)\end{array}$ & 0.122 \\
\hline \#24D & 18 & $\begin{array}{l}-0.04 \\
(0.66)\end{array}$ & 13 & $\begin{array}{c}-0.36 \\
(0.92)\end{array}$ & 0.470 & 12 & $\begin{array}{c}0.19 \\
(0.61)\end{array}$ & 11 & $\begin{array}{l}-0.03 \\
(1.15)\end{array}$ & 0.926 & 18 & $\begin{array}{l}-0.33 \\
(1.04)\end{array}$ & 12 & $\begin{array}{c}0.03 \\
(0.39)\end{array}$ & 0.339 \\
\hline \#25M & 17 & $\begin{array}{l}-0.42 \\
(1.55)\end{array}$ & 13 & $\begin{array}{c}0.08 \\
(0.86)\end{array}$ & 0.502 & 11 & $\begin{array}{c}-0.04 \\
(0.71)\end{array}$ & 10 & $\begin{array}{c}0.20 \\
(0.96)\end{array}$ & 0.502 & 19 & $\begin{array}{l}-0.14 \\
(1.07)\end{array}$ & 11 & $\begin{array}{c}0.17 \\
(0.54)\end{array}$ & 0.289 \\
\hline \#25D & 17 & $\begin{array}{l}-0.40 \\
(1.33)\end{array}$ & 13 & $\begin{array}{c}0.07 \\
(0.71)\end{array}$ & 0.130 & 11 & $\begin{array}{l}-0.28 \\
(0.68)\end{array}$ & 10 & $\begin{array}{l}-0.47 \\
(2.24)\end{array}$ & 0.306 & 19 & $\begin{array}{l}-0.21 \\
(0.95)\end{array}$ & 11 & $\begin{array}{c}0.11 \\
(0.73)\end{array}$ & 0.342 \\
\hline \#26M & 18 & $\begin{array}{c}0.39 \\
(1.69)\end{array}$ & 10 & $\begin{array}{c}0.10 \\
(0.65)\end{array}$ & 0.386 & 12 & $\begin{array}{l}-0.21 \\
(0.74)\end{array}$ & 10 & $\begin{array}{c}0.72 \\
(0.96)\end{array}$ & $0.032 *$ & 18 & $\begin{array}{c}0.27 \\
(0.75)\end{array}$ & 11 & $\begin{array}{c}0.26 \\
(1.18)\end{array}$ & 0.557 \\
\hline$\# 26 \mathrm{D}$ & 18 & $\begin{array}{c}0.53 \\
(1.86)\end{array}$ & 10 & $\begin{array}{c}0.25 \\
(0.56)\end{array}$ & 0.665 & 12 & $\begin{array}{c}0.26 \\
(1.94)\end{array}$ & 10 & $\begin{array}{c}0.11 \\
(1.33)\end{array}$ & 0.575 & 18 & $\begin{array}{c}0.11 \\
(1.14)\end{array}$ & 11 & $\begin{array}{c}0.05 \\
(0.65)\end{array}$ & 0.840 \\
\hline \#27M & 12 & $\begin{array}{c}0.93 \\
(1.85)\end{array}$ & 10 & $\begin{array}{l}-0.18 \\
(0.99)\end{array}$ & 0.261 & 10 & $\begin{array}{c}1.14 \\
(3.03)\end{array}$ & 10 & $\begin{array}{l}-0.27 \\
(2.42)\end{array}$ & 0.384 & 16 & $\begin{array}{c}0.79 \\
(3.59)\end{array}$ & 11 & $\begin{array}{c}0.56 \\
(0.98)\end{array}$ & 0.236 \\
\hline \#27D & 12 & $\begin{array}{c}0.09 \\
(1.73)\end{array}$ & 10 & $\begin{array}{l}-0.11 \\
(0.80)\end{array}$ & 0.766 & 10 & $\begin{array}{l}-0.04 \\
(1.00)\end{array}$ & 10 & $\begin{array}{c}0.12 \\
(0.76)\end{array}$ & 0.705 & 16 & $\begin{array}{c}0.28 \\
(1.33)\end{array}$ & 11 & $\begin{array}{c}0.41 \\
(1.26)\end{array}$ & 0.225 \\
\hline \multicolumn{16}{|l|}{ Site \#30 } \\
\hline \#34M & 10 & $\begin{array}{c}-0.43 \\
(0.72)\end{array}$ & 7 & $\begin{array}{c}0.16 \\
(0.49)\end{array}$ & 0.095 & 13 & $\begin{array}{c}0.16 \\
(0.50)\end{array}$ & 12 & $\begin{array}{c}0.13 \\
(0.79)\end{array}$ & 0.445 & 10 & $\begin{array}{c}0.38 \\
(1.47)\end{array}$ & 12 & $\begin{array}{c}0.30 \\
(0.59)\end{array}$ & 0.947 \\
\hline \#34D & 10 & $\begin{array}{l}-0.11 \\
(0.40)\end{array}$ & 7 & $\begin{array}{c}0.26 \\
(0.32)\end{array}$ & 0.077 & 13 & $\begin{array}{c}0.15 \\
(0.64)\end{array}$ & 12 & $\begin{array}{c}0.08 \\
(0.50)\end{array}$ & 0.784 & 10 & $\begin{array}{c}0.06 \\
(1.00)\end{array}$ & 12 & $\begin{array}{c}0.13 \\
(0.81)\end{array}$ & 0.869 \\
\hline \#35M & 10 & $\begin{array}{c}0.05 \\
(0.22)\end{array}$ & 8 & $\begin{array}{c}-0.05 \\
(0.78)\end{array}$ & 0.591 & 15 & $\begin{array}{c}-0.28 \\
(0.71)\end{array}$ & 12 & $\begin{array}{c}0.33 \\
(0.65)\end{array}$ & $0.042 *$ & 10 & $\begin{array}{l}-0.41 \\
(1.53)\end{array}$ & 11 & $\begin{array}{c}0.16 \\
(0.66)\end{array}$ & 0.191 \\
\hline \#35D & 10 & $\begin{array}{l}-0.13 \\
(0.63)\end{array}$ & 8 & $\begin{array}{c}0.29 \\
(0.49)\end{array}$ & 0.180 & 15 & $\begin{array}{c}0.31 \\
(0.56)\end{array}$ & 12 & $\begin{array}{c}-0.01 \\
(0.91)\end{array}$ & 0.261 & 10 & $\begin{array}{l}-0.33 \\
(1.20)\end{array}$ & 11 & $\begin{array}{l}-0.03 \\
(0.86)\end{array}$ & 0.572 \\
\hline \#36M & 11 & $\begin{array}{l}-0.18 \\
(0.81)\end{array}$ & 6 & $\begin{array}{l}-0.10 \\
(1.11)\end{array}$ & 0.480 & 14 & $\begin{array}{l}-0.10 \\
(0.49)\end{array}$ & 11 & $\begin{array}{l}-0.47 \\
(0.92)\end{array}$ & 0.364 & 10 & $\begin{array}{l}-0.25 \\
(1.25)\end{array}$ & 10 & $\begin{array}{c}0.55 \\
(0.70)\end{array}$ & 0.049 * \\
\hline \#36D & 11 & $\begin{array}{c}0.13 \\
(0.78)\end{array}$ & 6 & $\begin{array}{c}0.22 \\
(0.59)\end{array}$ & 0.920 & 14 & $\begin{array}{l}-0.21 \\
(0.82)\end{array}$ & 11 & $\begin{array}{c}0.42 \\
(1.23)\end{array}$ & 0.198 & 10 & $\begin{array}{c}0.20 \\
(1.20)\end{array}$ & 10 & $\begin{array}{c}0.24 \\
(0.85)\end{array}$ & 0.677 \\
\hline \#37M & 9 & $\begin{array}{c}0.63 \\
(1.62)\end{array}$ & 5 & $\begin{array}{l}-0.30 \\
(0.96)\end{array}$ & 0.255 & 15 & $\begin{array}{c}0.01 \\
(1.35)\end{array}$ & 11 & $\begin{array}{l}-0.02 \\
(0.82)\end{array}$ & 0.856 & 10 & $\begin{array}{c}0.18 \\
(1.36)\end{array}$ & 11 & $\begin{array}{c}0.90 \\
(1.66)\end{array}$ & 0.307 \\
\hline \#37D & 9 & $\begin{array}{l}-0.74 \\
(2.63)\end{array}$ & 5 & $\begin{array}{c}0.14 \\
(0.77)\end{array}$ & 0.503 & 15 & $\begin{array}{c}0.41 \\
(1.11)\end{array}$ & 11 & $\begin{array}{c}0.38 \\
(1.54)\end{array}$ & 0.795 & 10 & $\begin{array}{l}-0.53 \\
(1.71)\end{array}$ & 11 & $\begin{array}{c}0.21 \\
(1.14)\end{array}$ & 0.230 \\
\hline \multicolumn{16}{|l|}{ Site \#40 } \\
\hline$\# 44 \mathrm{M}$ & 14 & $\begin{array}{c}0.01 \\
(0.55)\end{array}$ & 11 & $\begin{array}{c}-0.04 \\
(0.30)\end{array}$ & 0.659 & 9 & $\begin{array}{l}-0.64 \\
(1.41)\end{array}$ & 13 & $\begin{array}{c}0.58 \\
(1.42)\end{array}$ & 0.116 & 13 & $\begin{array}{l}-0.10 \\
(1.05)\end{array}$ & 12 & $\begin{array}{c}-0.28 \\
(0.58)\end{array}$ & 0.785 \\
\hline \#44D & 14 & $\begin{array}{c}0.06 \\
(0.53)\end{array}$ & 11 & $\begin{array}{l}-0.02 \\
(0.53)\end{array}$ & 0.783 & 9 & $\begin{array}{l}-0.44 \\
(0.92)\end{array}$ & 13 & $\begin{array}{l}-0.06 \\
(0.76)\end{array}$ & 0.547 & 13 & $\begin{array}{c}0.90 \\
(1.74)\end{array}$ & 12 & $\begin{array}{c}0.07 \\
(0.51)\end{array}$ & 0.091 \\
\hline$\# 45 \mathrm{M}$ & 14 & $\begin{array}{c}0.14 \\
(0.95)\end{array}$ & 10 & $\begin{array}{c}0.03 \\
(0.70)\end{array}$ & 0.837 & 9 & $\begin{array}{l}-0.24 \\
(0.81)\end{array}$ & 14 & $\begin{array}{l}-0.06 \\
(0.84)\end{array}$ & 0.924 & 12 & $\begin{array}{c}0.48 \\
(0.63)\end{array}$ & 13 & $\begin{array}{c}0.28 \\
(1.25)\end{array}$ & 0.978 \\
\hline$\# 45 \mathrm{D}$ & 14 & $\begin{array}{l}-0.11 \\
(0.86)\end{array}$ & 10 & $\begin{array}{c}0.61 \\
(1.74)\end{array}$ & 0.394 & 9 & $\begin{array}{c}-0.08 \\
(0.48)\end{array}$ & 14 & $\begin{array}{c}0.03 \\
(0.61)\end{array}$ & 0.567 & 12 & $\begin{array}{c}0.13 \\
(0.53)\end{array}$ & 13 & $\begin{array}{c}0.21 \\
(0.93)\end{array}$ & 0.585 \\
\hline \#46M & 14 & $\begin{array}{l}-0.34 \\
(1.01)\end{array}$ & 11 & $\begin{array}{c}0.42 \\
(1.39)\end{array}$ & 0.227 & 8 & $\begin{array}{c}0.33 \\
(1.29)\end{array}$ & 11 & $\begin{array}{c}0.15 \\
(1.07)\end{array}$ & 1.000 & 12 & $\begin{array}{c}0.77 \\
(1.81)\end{array}$ & 12 & $\begin{array}{l}-0.16 \\
(1.59)\end{array}$ & 0.339 \\
\hline \#46D & 14 & $\begin{array}{l}-0.06 \\
(1.00)\end{array}$ & 11 & $\begin{array}{c}0.27 \\
(1.50)\end{array}$ & 0.913 & 8 & $\begin{array}{l}-0.01 \\
(0.92)\end{array}$ & 12 & $\begin{array}{c}0.17 \\
(1.32)\end{array}$ & 0.817 & 12 & $\begin{array}{c}0.03 \\
(0.65)\end{array}$ & 12 & $\begin{array}{c}0.29 \\
(0.76)\end{array}$ & 0.418 \\
\hline$\# 47 \mathrm{M}$ & 13 & $\begin{array}{l}-0.25 \\
(0.95)\end{array}$ & 8 & $\begin{array}{c}0.21 \\
(0.49)\end{array}$ & 0.076 & 7 & $\begin{array}{l}-1.14 \\
(2.01)\end{array}$ & 12 & $\begin{array}{c}0.36 \\
(2.11)\end{array}$ & 0.117 & 13 & $\begin{array}{l}-0.07 \\
(0.97)\end{array}$ & 9 & $\begin{array}{c}0.86 \\
(1.44)\end{array}$ & 0.150 \\
\hline \#47D & 13 & $\begin{array}{c}-0.34 \\
(2.48)\end{array}$ & 8 & $\begin{array}{c}0.06 \\
(0.61)\end{array}$ & 0.514 & 7 & $\begin{array}{l}-0.87 \\
(1.42)\end{array}$ & 12 & $\begin{array}{l}-0.01 \\
(2.93)\end{array}$ & 0.525 & 13 & $\begin{array}{c}0.18 \\
(2.53)\end{array}$ & 9 & $\begin{array}{c}0.66 \\
(1.66)\end{array}$ & 0.332 \\
\hline
\end{tabular}

$p: p$ values among subjects in the GTR or Flap groups (*: Statistical significance level was 5\%, $p<0.05)$. GTR: Guided tissue regeneration. \#14-17: Right upper premolar and molar region (FDI numbering system). \#24-27: Left upper premolar and molar region (FDI numbering system). \#34-37: Left lower premolar and molar region (FDI numbering system). \#44-47: Right lower premolar and molar region (FDI numbering system).

\subsection{Multivariable Analysis for Alveolar Bone Loss after Treatment}

In multivariable analysis for alveolar bone loss after treatment, the type of surgery and upper/lower jaw variables were significantly associated with alveolar bone loss (odds ratio (OR), $0.731 ; 95 \%$ confidence interval $(\mathrm{CI}), 0.619-0.862 ; p=0.001$; type of surgery 
vs. odds ratio (OR), 1.255; 95\% confidence interval (CI), 1.063-1.481; upper/lower jaw, $p=0.007$ ) (Table 7).

Table 7. Multivariable analysis association between alveolar bone loss and other variables after treatment.

\begin{tabular}{cccc}
\hline & \multicolumn{3}{c}{ Alveolar Bone Loss } \\
\cline { 2 - 4 } & OR & $\mathbf{9 5 \% ~ C I ~}$ & $p$ \\
\hline Type of surgery (GTR/Flap) & 0.731 & $0.619-0.862$ & $0.001^{\text {* }}$ \\
Sex & 1.025 & $0.849-1.237$ & 0.798 \\
Age & 0.998 & $0.988-1.009$ & 0.753 \\
Smoking & 0.979 & $0.790-1.214$ & 0.847 \\
Systemic diseases & 1.275 & $0.794-2.049$ & 0.354 \\
Medicine & 1.295 & $0.804-2.085$ & 0.514 \\
Upper/Lower jaw & 1.255 & $1.063-1.481$ & $0.007^{*}$ \\
Sites & 1.055 & $0.832-1.336$ & 0.528 \\
\hline
\end{tabular}

Multivariable analysis using logistic regression model: type of surgery, sex, age, smoking, systemic disease, medicine, upper/lower jaw, and precise sites (*: Statistical significance level was $5 \%, p<0.05)$.

\section{Discussion}

The present study showed that the change of bone height after GTR and after access flap alone procedures showed differences. Our findings indicate that the bone height of several teeth in the GTR group was significantly higher compared with those teeth in the Flap group (\#14D, \#15M, and \#47M). Females in the GTR group had significantly more favorable values than males. In males, $\# 47$ showed significant healing of bone height. Regarding smoking, there was notable bone loss in the GTR group among smokers. All patients were divided by three age-related subgroups consisting of those $29-45,46-53$, and 54-76 years old. At lower ages (29-45), the GTR group showed less bone resorption than the Flap group. The smaller the interval between before and after $\mathrm{CBCT}$, the more bone increase and the less bone resorption. It was observed that the smaller the interval between shots of $\mathrm{CBCT}$, the less bone resorption. In particular, in the absence of systemic diseases or no medications, the GTR group showed less bone resorption and better healing than the Flap group. A multivariable analysis suggested that the type of surgery and surgical site (upper/lower jaw) were associated with bone resorption after treatment.

In this study, the comparison between groups showed less alveolar bone loss and superior bone healing in the GTR group vs. the Flap group as a whole. The same result was seen in a previous study. Tonetti et al. suggested that regenerative periodontal surgery with GTR had PPD reduction and CAL gain [20]. They also noted that the absolute value of the added healing portion was relatively small, but it was in agreement with other similar research studies [22,23]. In a systemic review of 13 articles, regenerative surgeries have shown an adjunctive benefit of CAL gain [24]. A distinctive point observed in this study was that 28 teeth were extracted only from the Flap group during the follow-up period after periodontal surgery. It seems that the GTR procedure is a superior treatment to prolong tooth life span compared with the Flap procedure. Long-term study of GTR vs. open flap reported that there was no significant difference of bone loss between GTR and open Flap surgery [21]. The present study revealed that GTR in the mandible yielded better bone gain than in the maxilla. These outcomes are similar to those reported by Odontuya et al. [25]. Other researchers explained that this difference was due to the complex morphology and accessibility of molars such as root cuvature and furcations [26,27].

This study used DFDBA in combination with resorbable membrane in the GTR group. DFDBA is an allograft with potential osteoinductive ability to expose BMPs that promote osteoblast differentiation, and it would be beneficial for bone regeneration $[28,29]$. Camelo et al. demonstrated that DFDBA with collagen membrane had better clinical outcomes of bony healing in a human study [30]. In an animal study, the combination of membrane and DFDBA has been proven to regenerate bone [31]. By demineralized processing, DFDBA has higher osteoinductivity but lower mechanical property. Bone graft material should prevent the resorbable membrane from moving downward. For this 
reason, physical strength is necessary. DFDBA has relatively weaker mechanical properties than freeze-dried bone allograft (FDBA) or xenograft, and it might affect results when used clinically. A previous study failed to find a difference between bone grafting with DFDBA and a no-bone grafting procedure [32].

Resorbable membranes have been introduced as a barrier to prevent moving epithelium apically [33]. While resorbable membranes have many favorable aspects, they have major limitations, including weaker physical strength [34]. A previous study found that it takes approximately 4-24 weeks for the degradation of collagen membranes [11]. Crosslinked membranes have been developed to increase the stiffness and delay the degradation of resorbable membranes and have shown superior physical strength relative to non-crosslinked membranes [13,35]. Cross-linked membranes were used in this study. In a previous study, the application of resorbable membranes was favorable at infrabony defects [32]. Another study demonstrated that combining bone graft material with resorbable membrane resulted in more benefit than only membrane [36]. However, Stavropoulos et al. did not find any difference between those two methods (bone + membrane vs. membrane only) [6]. In this study, porcine collagen was used as the source of resorbable membranes. Bovine collagen is one of the major sources of resorbable membranes, but the drawback of this collagen is the associated allergic reactions [37]. Porcine collagen in a known alternative to bovine collagen. A previous study found that porcine collagen led to minimal allergic reactions because porcine collagen seemed more similar to human collagen [38]. Lee et al. reported a similar clinical expediency and properties when comparing porcine cross-linked vs. non-cross-linked collagen membrane [39].

This study differs from previous studies in that it compared the situation before and after treatment using CBCT. The detection of periodontal bone loss is mandatory for accurate diagnosis. Clinical methods including probing have shown limitations in reliability. Various factors such as the probing force, shape of the tip, and direction of the probe affect the clinical results. In addition, it is not easy to accurately assess the healing point due to resistance of tissue [40]. It is difficult to get an accurate image using overlapping images from conventional radiographs such as intraoral periapical and panoramic radiographs. CBCT has been introduced to overcome the drawbacks of conventional radiographic methods. CBCT can get a specific cross-sectional image and can reconstruct radiographic images with a multi-dimensional view [16-18]. There were several previous studies in which CBCT was used to measure the amount of bone loss in periodontitis. Mohan et al. reported that there was no difference in bone loss between CBCT and actual measurement of surgically exposed osseous defects [40]. In addition, CBCT was useful for identifying buccal and lingual bony defects in aggressive periodontitis. Another study has concluded that CBCT had the ability to assess the maxillary or mandibular furcation area [41]. CBCT can identify the root concavities of premolars and bone loss pattern [42]. An important advantage of CBCT is the low radiation dose. The radiation of CBCT has been reported to be 15 times lower than conventional radiography [43].

The limitation of this restorative study is that the conditions such as systemic disease and oral environment of the test and control group could not be perfectly matched. To enhance these drawback, future studies will investigate prospective research for the controlled subjects. In addition, other bone substitutes including xenograft and alloplast might be suggested to use for further research. A strength of the present study is that a large number of subjects using $\mathrm{CBCT}$ were involved. The design of this study differs from previous studies in that it directly observed bone changes using CBCT. PPD and CAL may be good clinical indicators. However, radiologic measurement with CBCT is also good and accurate for evaluating the effectiveness of periodontal treatment.

\section{Conclusions}

Despite the limitations of this study, the results suggest that GTR with bone grafting can be a more effective method for bone healing and delay the extraction of teeth than Flap alone. 
Author Contributions: Conceptualization, J.-T.L.; formal analysis, J.-T.L. and H.-S.C.; investigation, J.-T.L., H.-S.C. and I.-K.L.; methodology, S.-H.J. and J.-T.L.; project administration, J.-T.L.; writingoriginal draft, I.-K.L. and J.-T.L.; writing-review and editing, J.-T.L. All authors have read and agreed to the published version of the manuscript.

Funding: This work was funded by Hyundai bioland Co., Ltd., Cheongju, Korea (grant number: 1810-001-002).

Institutional Review Board Statement: The study was conducted according to the guidelines of the Declaration of Helsinki and approved by the Institutional Review Board for Clinical Research at Dankook University College of Dentistry Jukjeon Dental Hospital (approval no. 201910-001-002; approved date: 17 October 2019).

Informed Consent Statement: Not applicable.

Data Availability Statement: All available data are presented within the article or are available on request from the corresponding author.

Conflicts of Interest: The authors declare no conflict of interest.

\section{References}

1. Lang, N.P.; Lindhe, J. Clinical Periodontology and Implant Dentistry, 5th ed.; John Wiley \& Sons: New York, NY, USA, 2015; pp. 783-786.

2. Rosen, P.S.; Reynolds, M.A.; Bowers, G.M. The treatment of intrabony defects with bone grafts. J. Periodontol. 2000, 22, 88-103. [CrossRef]

3. Cortellini, P.; Tonetti, M.S. Focus on intrabony defects: Guided tissue regeneration. Periodontol. 2000 2000, 22, 104-132. [CrossRef]

4. Nevins, M.L.; Camelo, M.; Lynch, S.E.; Schenk, R.K.; Nevins, M. Evaluation of periodontal regeneration following grafting intrabony defects with bio-oss collagen: A human histologic report. Int. J. Periodontics Restor. Dent. 2003, $23,9-17$.

5. Sculean, A.; Berakdar, M.; Chiantella, G.C.; Donos, N.; Arweiler, N.B.; Brecx, M. Healing of intrabony defects following treatment with a bovine-derived xenograft and collagen membrane: A controlled clinical study. J. Periodontol. 2003, 30, 73-80. [CrossRef] [PubMed]

6. Stavropoulos, A.; Karring, E.S.; Kostopoulos, L.; Karring, T. Deproteinized bovine bone and gentamicin as an adjunct to GTR in the treatment of intrabony defects: A randomized controlled clinical study. J. Clin. Periodontol. 2003, 30, 486-495. [CrossRef]

7. Blank, B.S.; Levy, A.R. Combined treatment of a large periodontal defect using GTR and DFDBA. J. Int. J. Periodontics Restor. Dent. 1999, 19, 480-487.

8. Parashis, A.; Andronikaki-Faldami, A.; Tsiklakis, K. Comparison of 2 Regenerative Procedures-Guided Tissue Regeneration and Demineralized Freeze-Dried Bone Allograft-in the Treatment of Intrabony Defects: A Clinical and Radiographic Study. J. Periodontol. 1998, 69, 751-758. [CrossRef]

9. Luepke, P.G.; Mellonig, J.T.; Brunsvold, M.A. A clinical evaluation of a bioresorbable barrier with and without decalcified freeze-dried bone allograft in the treatment of molar furcations. J. Clin. Periodontol. 1997, 24, 440-446. [CrossRef]

10. Kiany, F.; Moloudi, F. Amnion membrane as a novel barrier in the treatment of intrabony defects: A controlled clinical trial. Int. J. Oral. Maxillofac. Implant. 2015, 30, 639-647. [CrossRef]

11. Bunyaratavej, P.; Wang, H.L. Collagen membranes: A review. J. Periodontol. 2001, 72, 215-229. [CrossRef] [PubMed]

12. Sanz-Sánchez, I.; Ortiz-Vigón, A.; Sanz-Martin, I.; Figuero, E.; Sanz, M. Effectiveness of lateral bone augmentation on the alveolar crest dimension: A systematic review and meta-analysis. Dent. Res. J. 2015, 94, 128S-142S. [CrossRef] [PubMed]

13. Behring, J.; Junker, R.; Walboomers, X.F.; Chessnut, B.; Jansen, J.A. Toward guided tissue and bone regeneration: Morphology, attachment, proliferation, and migration of cells cultured on collagen barrier membranes. A systematic review. Odontology 2008, 96, 1-11. [CrossRef] [PubMed]

14. Becker, J.; Al-Nawas, B.; Klein, M.O.; Schliephake, H.; Terheyden, H.; Schwarz, F. Use of a new cross-linked collagen membrane for the treatment of dehiscence-type defects at titanium implants: A prospective, randomized-controlled double-blinded clinical multicenter study. Clin. Oral. Implant. Res. 2009, 20, 742-749. [CrossRef]

15. Drexler, J.W.; Powell, H.M. Dehydrothermal crosslinking of electrospun collagen. Tissue Eng. Part C Methods 2011, 17, 9-17. [CrossRef] [PubMed]

16. Misch, K.A.; Yi, E.S.; Sarment, D.P. Accuracy of cone beam computed tomography for periodontal defect measurements. J. Periodontol. 2006, 77, 1261-1266. [CrossRef] [PubMed]

17. Vandenberghe, B.; Jacobs, R.; Yang, J. Diagnostic validity (or acuity) of 2D CCD versus 3D CBCT-images for assessing periodontal breakdown. Oral. Surg. Oral. Med. Oral. Pathol. Oral. Radiol. 2007, 104, 395-401. [CrossRef] [PubMed]

18. Vandenberghe, B.; Jacobs, R.; Yang, J. Detection of periodontal bone loss using digital intraoral and cone beam computed tomography images: An in vitro assessment of bony and/or infrabony defects. Dentomaxillofac. Radiol. 2008, 37, 252-260. [CrossRef] 
19. Ratka-Krüger, P.; Neukranz, E.; Raetzke, P. Guided tissue regeneration procedure with bioresorbable membranes versus conventional flap surgery in the treatment of infrabony periodontal defects. J. Clin. Periodontol. 2000, 27, 120-127. [CrossRef]

20. Tonetti, M.S.; Cortellini, P.; Lang, N.P.; Suvan, J.E.; Adriaens, P.; Dubravec, D. Clinical outcomes following treatment of human intrabony defects with GTR/bone replacement material or access flap alone. A multicenter randomized controlled clinical trial. J. Clin. Periodontol. 2004, 31, 770-776. [CrossRef]

21. Petsos, H.; Ratka-Krüger, P.; Neukranz, E.; Raetzke, P.; Eickholz, P.; Nickles, K. Infrabony defects 20 years after open flap debridement and guided tissue regeneration. J. Clin. Periodontol. 2019, 46, 552-563. [CrossRef]

22. Tonetti, M.; Cortellini, P.; Suvan, J.; Adriaens, P.; Baldi, C.; Dubravec, D. Evaluation of generalizability of the added benefit of GTR in the treatment of deep intrabony defects. A phase iv multicenter randomized controlled clinical trial. J. Periodontol. 1998, 69, 1183-1192. [CrossRef]

23. Cortellini, P.; Tonetti, M.S.; Lang, N.P.; Suvan, J.E.; Zucchelli, G.; Vangsted, T. The simplified papilla preservation flap in the regenerative treatment of deep intrabony defects: Clinical outcomes and postoperative morbidity. J. Periodontol. 2001, 72, 1702-1712. [CrossRef]

24. Nibali, L.; Koidou, V.P.; Nieri, M.; Barbato, L.; Pagliaro, U.; Cairo, F. Regenerative surgery versus access flap for the treatment of intrabony periodontal defects. A systematic review and meta-analysis. J. Clin. Periodontol. 2019, 47, 320-351. [CrossRef]

25. Dorj, O.; Lee, W.-F.; Salamanca, E.; Pan, Y.-H.; Wu, Y.-F.; Hsu, Y.-S.; Lin, J.C.Y.; Lin, Y.-D.; Choy, C.-S.; Chang, W.-J. Guided Tissue Regeneration Treatment Yields Better Results in Class II Furcations in the Mandible Than in the Maxilla: A Retrospective Study. Int. J. Environ. Res. Public Health 2021, 18, 7447. [CrossRef]

26. Metzler, D.G.; Seamons, B.C.; Mellonig, J.T.; Gher, M.E.; Gray, J.L. Clinical Evaluation of Guided Tissue Regeneration in the Treatment of Maxillary Class II Molar Furcation Invasions. J. Periodontol. 1991, 62, 353-360. [CrossRef]

27. Pontoriero, R.; Lindhe, J. Guided tissue regeneration in the treatment of degree II furcations in maxillary molars. J. Clin. Periodontol. 1995, 22, 756-763. [CrossRef]

28. Bhedasgaonkar, S.Y.; Kapadia, J.; Patil, N.A. Treatment of infrabony defects with platelet-rich fibrin along with bone graft: Case report. J. Int. Clin. Dent. Res. Organ. 2015, 7, 69-74.

29. Hills, D. Evaluation of Demineralized Freeze-Dried Bone Allograft in Combination with Chorion Membrane in the Treatment of Grade II Furcation Defects: A Randomized Controlled Trial. Periodontics 2019, 39, 659-667.

30. Camelo, M.; Nevins, M.L.; Lynch, S.E.; Schenk, R.K.; Simion, M.; Nevins, M. Periodontal regeneration with an autogenous bone-Bio-Oss composite graft and a Bio-Gide membrane. Int. J. Periodontics Restor. Dent. 2001, 21, 109-120.

31. Yamada, S.; Shima, N.; Kitamura, H.; Sugito, H. Effect of porous xenographic bone graft with collagen barrier membrane on periodontal regeneration. Int. J. Periodontics Restor. Dent. 2002, 22, 389-397.

32. Murphy, K.G.; Gunsolley, J.C. Guided tissue regeneration for the treatment of periodontal intrabony and furcation defects. A systematic review. Ann. Periodontol. 2003, 8, 266-302. [CrossRef] [PubMed]

33. Ortolani, E.; Quadrini, F.; Bellisario, D.; Santo, L.; Polimeni, A.; Santarsiero, A. Mechanical qualification of collagen membranes used in dentistry. Ann. dell'Istituto Super. Sanita 2015, 51, 229-235.

34. Park, J.I.; Yang, C.; Kim, Y.T.; Kim, M.S.; Lee, J.S.; Choi, S.H. Space maintenance using crosslinked collagenated porcine bone grafted without a barrier membrane in one-wall intrabony defects. J. Biomed. Mater. Res. B Appl. Biomater. 2014, 102, $1454-1461$. [CrossRef] [PubMed]

35. Jung, U.W.; Cha, J.K.; Vignoletti, F.; Nuñez, J.; Sanz, J.; Sanz, M. Simultaneous lateral bone augmentation and implant placement using a particulated synthetic bone substitute around chronic peri-implant dehiscence defects in dogs. J. Clin. Periodontol. 2017, 44, 1172-1180. [CrossRef]

36. Paolantonio, M. Combined periodontal regenerative technique in human intrabony defects by collagen membranes and anorganic bovine bone. A controlled clinical study. J. Periodontol. 2002, 73, 158-166. [CrossRef]

37. Silvipriya, K.; Kumar, K.K.; Bhat, A.; Kumar, B.D.; John, A.; Lakshmanan, P. Collagen: Animal sources and biomedical application. J. Appl. Pharm. Sci. 2015, 5, 123-127. [CrossRef]

38. Cortial, D.; Gouttenoire, J.; Rousseau, C.; Ronzière, M.-C.; Piccardi, N.; Msika, P. Activation by IL-1 of bovine articular chondrocytes in culture within a $3 \mathrm{D}$ collagen-based scaffold. An in vitro model to address the effect of compounds with therapeutic potential in osteoarthritis. Osteoarthr. Cartil. 2006, 14, 631-640. [CrossRef]

39. Lee, J.H.; Lee, J.S.; Baek, W.S.; Lim, H.C.; Cha, J.K.; Choi, S.H. Assessment of dehydrothermally cross-linked collagen membrane for guided bone regeneration around peri-implant dehiscence defects: A randomized single-blinded clinical trial. J. Periodontal Implant. Sci. 2015, 45, 229-237. [CrossRef]

40. Mohan, R.; Mark, R.; Sing, I.; Jain, A. Diagnostic Accuracy of CBCT for Aggressive Periodontitis. J. Clin. Imaging Sci. 2014, 4, 2. [CrossRef]

41. Feijo, C.V.; Lucena, J.G.; Kurita, L.M.; Pereira, S.L. Evaluation of cone beam computed tomography in the detection of horizontal periodontal bone defects: An in vivo study. Int. J. Periodontics Restor. Dent. 2012, 32, 162-168.

42. Zhao, H.; Wang, H.; Pan, Y.; Pan, C.; Jin, X. The relationship between root concavities in first premolars and chronic periodontitis. J. Periodontal. Res. 2014, 49, 213-219. [CrossRef] [PubMed]

43. Ludlow, J.B.; Davies-Ludlow, L.E.; Brooks, S.L.; Howerton, W.B. Dosimetry of 3 CBCT devices for oral and maxillofacial radiology: CB Mercuray, NewTom 3G and i-CAT. Dentomaxillofac. Radiol. 2006, 35, 219-226. [CrossRef] [PubMed] 